\title{
手性单齿亚磷酸酯配体在不对称氢化反应中的研究进展
}

\author{
赵芳 $a$ 叶文静*,a,b 王 凯*,a \\ ( ${ }^{a}$ 湖北大学有机功能分子合成与应用教育部重点实验室 武汉 430062) \\ ( $b$ 湖北大学药物高通量篮选技术国家地方联合工程研究中心 武汉 430062)
}

\begin{abstract}
摘要 进入 21 世纪以来, 手性单磷配体特别是手性亚磷酸酯和亚磷酰胺类单磷配体的研究引起了国内外的广泛关注, 这些单齿磷配体的合成简单、原料价格低廉、性质稳定，同时在不对称催化反应中表现出高活性和对映选择性. 对近 二十年来不对称催化氢化领域里手性亚磷酸酯配体的发展历程进行了综述, 介绍了亚膦酸酯(Phosphonites)、亚磷酸酯 (Phosphites)和亚磷酰胺(Phosphoramidites)三类单齿磷配体以及由这三类配体衍生出的其他应用.
\end{abstract}

关键词 亚磷酸酯; 亚磷酰胺; 亚膦酸酯; 单齿配体; 不对称氢化

\section{Advancement of Chiral Monodentate Phosphite Ligands in Asymmetric Hydrogenation}

\author{
Zhao, Fang ${ }^{a} \quad$ Ye, Wenjing*,a,b $\quad$ Wang, Kai ${ }^{*, a}$ \\ ( ${ }^{a}$ Ministry-of-Education Key Laboratory for the Synthesis and Application of Organic Functional Molecule, \\ Hubei University, Wuhan 430062) \\ ( ${ }^{b}$ National \& Local Joint Engineering Research Center of High-Throughput Drug Screening Technology, \\ Hubei University, Wuhan 430062)
}

\begin{abstract}
Since the 21st century, the study of chiral monophosphorous ligands, especially chiral mono-phosphites ligands and mono-phosphoramidites ligands has attracted widespread attention. This kind of ligands was extensively used in various asymmetric catalytic reactions, for most of them were easily synthesized and showed good stability, high activity and high enantioselectivity. The diverse common monodentate phosphonites, phosphites, and phosphoramidites ligands and their applications in asymmetric hydrogenations over the past two decades are reviewed.
\end{abstract}

Keywords phosphites; phosphoramidites; phosphonites; monodentate ligands; asymmetric hydrogenation

在近 50 年深入研究中, 不对称氢化取得了巨大成 就, 一些不对称氢化反应成为实验室合成手性化合物的 常规反应，同时投入到制药、农学和香水等领域的工业 生产中 [1]. 手性含磷配体参与形成的过渡金属配合物催 化的不对称氢化是目前应用到实际中的较成功的例子 之一, 因该种配体对后过渡金属有很好的配位性及触发 催化剂催化活性的能力, 所以在不对称催化中得到了广 泛的应用 ${ }^{[2]}$.

根据配体结构分类，手性含磷配体可以分为单齿、 双齿和多齿类型. 在 20 世纪后叶, 手性磷配体的设计与 合成都聚焦于具有 $C_{2}$ 轴的双齿鳌合膦配体(dipho- sphines), 它被看作是有效不对称还原的前提条件. 21 世 纪初, Pringle ${ }^{[3]}$ 、 Reetz ${ }^{[4]}$ 和 Feringa ${ }^{[5]}$ 相继报道了亚膦酸酯 (phosphonites)、亚磷酸酯(phosphites)、亚磷酰胺(phosphorramidites)在不对称催化氢化中的应用, 发现对应 的催化体系表现不亚于双齿膦配体(diphosphines), 且这 三类配体合成简单，原料低廉. 自此, 关于这三类单齿 配体的报道取得了蓬勃的发展 ${ }^{[6-8]}$.

综述了自 2000 年来这三类单齿配体在不对称氢化 领域中取得的一些成果，常用的底物如 $\alpha$-乙酰氨基肉桂 酸甲酯 1、衣康酸二甲酯 $2 、 \alpha$-乙酰氨基丙烯酸甲酯 3 以及 $\alpha$-苯基烯酰胺 4 一般作为模型底物考察磷配体对反

\footnotetext{
* Corresponding authors. E-mail: wye@hubu.edu.cn; kaiwang@hubu.edu.cn

Received November 12, 2020; revised January 31, 2021; published online March 12, 2021.

Project supported by the Ministry of Education Key Laboratory for the Synthesis and Application of Organic Functional Molecules, Hubei University (No. KLSAOFM1910).

湖北大学有机功能分子合成与应用教育部重点实验室(No. KLSAOFM1910)资助项目.
} 
应活性和对映选择性的影响(图 1).

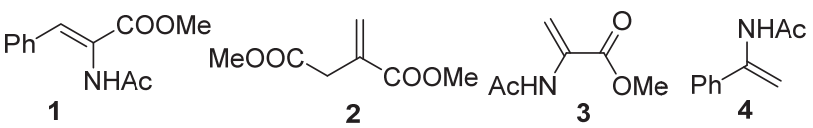

图 1 常见的催化氢化标准底物

Figure 1 Common benchmark substrates for asymmetric hydrogenation $(\mathrm{AH})$

\section{1 单齿磷配体的起源}

1965 年, Wilkinson 将 $\mathrm{RhCl}\left(\mathrm{PPh}_{3}\right)_{3}$ 用作催化剂实现 了温和条件下简单烯烃的催化氢化. 在他的启发之下, Knowles ${ }^{[9-10]}$ 和 Horner 等 ${ }^{[11]}$ 分别提出了将三苯基膦衍生 的手性配体应用到潜手性底物的不对称氢化中. 1968

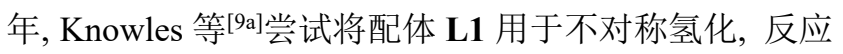
虽然发生，但对映选择性较低，ee 值最高仅 $15 \% .1972$ 年, Knowles 等 ${ }^{[96]}$ 设计出了配体 PAMP (L2) 和 CAMP (L3), 在 $\alpha$-脱氢氨基酸 $\mathbf{5}$ 的不对称氢化中, PAMP 配体诱 导的产物对映选择性较差(仅 $55 \% e e$ ), 而 CAMP 表现出 更好的手性诱导能力, 高达 $88 \%$ ee (Eq. 1). Knowles 推 测 $\mathbf{L 2}$ 的手性诱导能力不理想可能是由于形成的配合物 中 Rh-P 原子会自由旋转. 基于此, 他设计将 $\mathbf{L} 2$ 的两个 磷原子进行桥联得到双齿配体 di-PAMP (L4), 不对称诱 导能力高达 $95 \% e e^{[9 c]}$, 最终用于 $L$-DOPA 的大规模生 产，但该配体制备过程就当时的条件而言相当繁琐。 1971 年, Kagan 等 ${ }^{[12-13]}$ 设计了双齿配体 DIOP (L5), 该配 体的光学活性源于手性骨架而非 P 原子, 可由价格低廉 易得的酒石酸酯制得, 且合成步骤简单, 手性诱导活性 强, 由此, 开启了双齿膦配体的时代. 尽管 Knowles 等 ${ }^{[0]}$ 合成的单齿膦配体 CAMP 的立体诱导活性达到 $90 \%$, 但是在后面的 30 年内, 单齿磷配体一直没有引起 人们足够的重视.

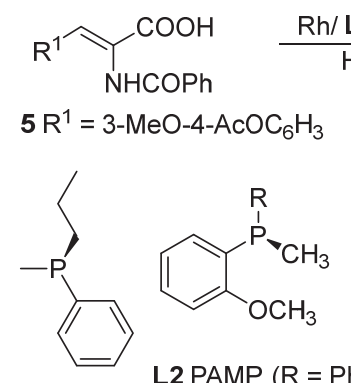

L1 L3 CAMP $(R=$ cy)
$\underset{\mathrm{H}_{2}}{\stackrel{\mathrm{Rh} / \text { L2, L3 }}{\longrightarrow}}$

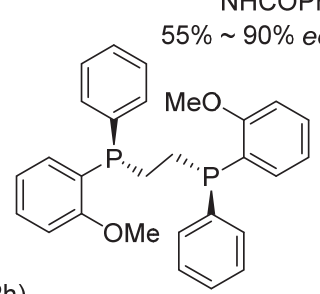

L4 di-PAMP
2000 年, 基于 BINOL 骨架的亚膦酸酯[3,14]、亚磷酸 酯 ${ }^{[4]}$ 、亚磷酰胺 ${ }^{[5,15]}$ 以优异的对映选择性成功应用于 $R h$ 催化烯烃的不对称氢化反应, 获得了可以媲美双齿配体 的催化效率和对映选择性. 合成这类配体的原料是廉价 兼具光活性的手性骨架 BINOL, 仅需 1 2 步反应即可 制得, 且无需手性拆分, 其成本至少比双齿膦配体便宜

一个数量级. 这几类合成简便、催化效率高、手性诱导 能力强的单齿磷配体的出现, 为开发高效的不对称氢化 催化体系创造了更多机会.

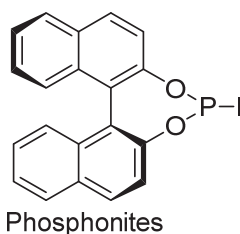

Phosphonites
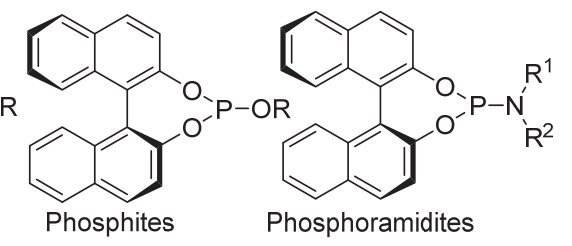

第一个发展机会是其在组合化学上的应用. 在不对 称氢化过程中，活性配合物通常含有两个单齿配体与金 属配位 ${ }^{[16]}$, 所以原则上可以制备出同时含有两个不同 单齿配体的金属复合物 $\mathrm{ML}_{\mathrm{a}} \mathrm{L}_{\mathrm{b}}{ }^{[17-21]}$. 但在溶液中配体交 换速度很快，最终会得到包含三种复合物的混合物 (Scheme 1), 其比例由配合物的热力学稳定性决定, 还 可通过改变配体的比例来调节 ${ }^{[20]}$. 多数情况下, $\mathrm{ML}_{a} \mathrm{~L}_{b}$ 的催化活性和选择性优于 $\mathrm{ML}_{a} \mathrm{~L}_{a}$ 或 $\mathrm{ML}_{b} \mathrm{~L}_{b}$, 所以控制溶 液中三种配合物的平衡向 $\mathrm{ML}_{\mathrm{a}} \mathrm{L}_{\mathrm{b}}$ 发展是很关键的, 有三 种方法可以使用. (一)可以在配体上引入选择性识别单 元促进两种配体自组装形成双齿形式的超分子配

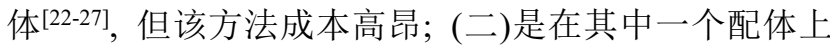
引入阴离子基团，由于电荷排斥，平衡会往异种组合配 合物(MNA)的方向移动(Scheme 2a); (三)使用磷酸二酯 配体(氢键给体)和亚磷酰胺配体 (氢键受体)形成一个含 分子内氢键的异种组合复合物(Scheme 2b). 2013 年, Pfaltz 等[28]将含阴离子基团的亚磷酰胺配体 L6 与中性 磷酸二酯配体 L7 或 L8 的异种组合成功应用于烯酰胺 6 的不对称氢化中 (Scheme 2c). 机理研究表明, 两个配体 间氢键的形成稳定了异种组合催化剂, 所以反应的立体 选择性很优异.

$$
\begin{aligned}
& L_{a}+L_{b} \stackrel{M}{\longrightarrow} M L_{a} L_{b}+M L_{a} L_{a}+M L_{b} L_{b} \\
& 2 M L_{a} L_{b} \rightleftharpoons M L_{a} L_{a}+M L_{b} L_{b}
\end{aligned}
$$

图式 1 二元混合配体配合物的平衡关系

Scheme 1 Equilibrium relationship between the complexes of binary hybrid ligands

其次，单齿配体的易合成性为不对称氢化的发展提 供了另一个机会: 配体的快速平行合成和高通量篮选 $(\mathrm{HTS})^{[29]}$. 该方法由荷兰的 Lefot 开发出来, 利用单齿亚 磷酰胺配体合成步骤简单、原料低廉的优点, 平行制备 多个亚磷酰胺配体，并将平行合成的一系列配体与金属 催化前体混合, 用于不饱和底物的不对称氢化中, 可快 速筛选出最佳催化体系. 该方法显著缩短了寻找与不同 底物匹配的高催化活性和高诱导能力配体的时间，被迅 速应用于实验室篮选配体和工业生产中, 有力推动了不 对称催化的发展. 2010 年, Lefort 等[30]利用 HTS 找到可 催化大位阻芳基烯酰胺的最佳配体，用来大量合成一种 


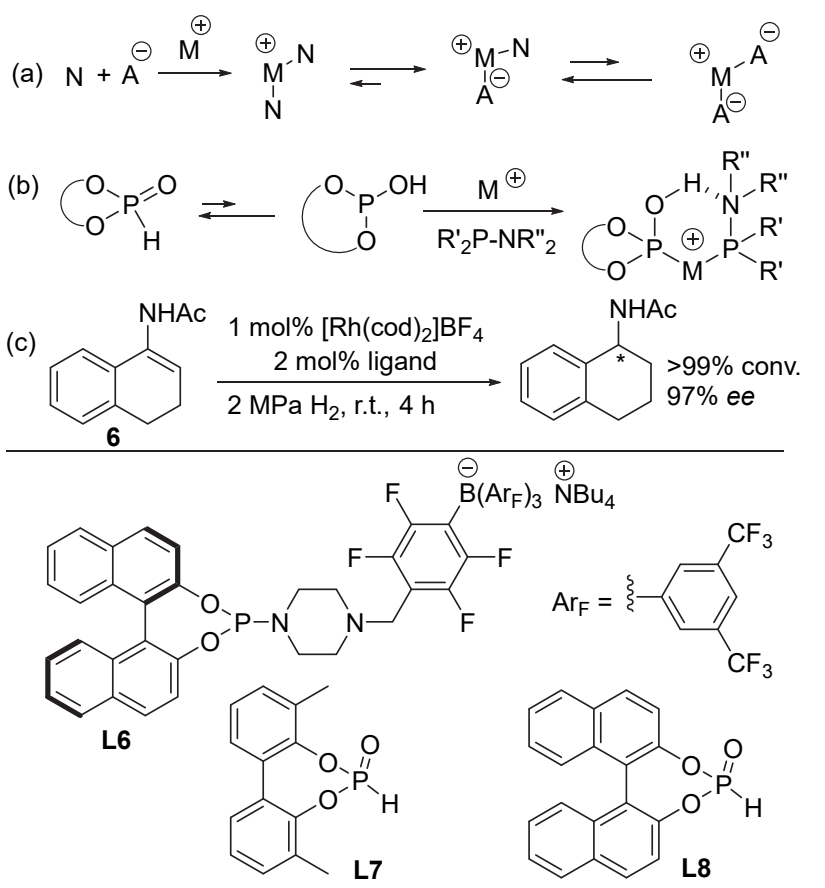

图式 2 反应平衡向二元杂合配体方向进行的方法及其在不 对称氢化中的应用

Scheme 2 Method for reaction equilibrium to proceed towards binary hybrid ligand and their application in $\mathrm{AH}$

治疗肥胖症的药物中间体. 从小试到将反应放大至千克 级量产，前后仅花费约 $25 \mathrm{~d}$ 时间.

BINOL 骨架取得的成功, 也促进了其他类型手性

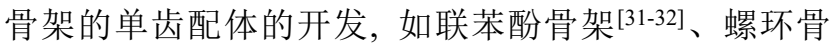

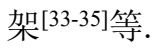

\section{2 单齿亚膦酸酯配体(Phosphonites)}

在不对称催化中, 亚膦酸酯配体是一类很有价值的 配体, 与芳基单膦配体(mono arylphosphines)相比, 单齿 亚膦酸酯配体中与 $\mathrm{P}$ 原子相连的烷氧基是吸电子基, 因 此 $\mathrm{P}$ 原子处于缺电子状态, 其 $\sigma$ 给体弱但 $\pi$ 受体强, $\sigma^{*}$ 轨道能量降低, 这样的特性使得该类配体可以更好的稳 定低氧化态的过渡金属 ${ }^{[36]}$ 。

2000 年, Pringle 等 ${ }^{[3]}$ 报道了单齿亚膦酸酯配体 $\mathbf{L 9 a} \sim \mathbf{L 9 c}$ (图 2). 将配体对应的 Rh 配合物催化 $\alpha$-乙酰氨 基丙烯酸甲酯 3 的不对称氢化反应, 其中 L9a 对应可得 $92 \% e e$ 的氢化产物, 但是转化率稍低, 仅 $73 \%$ (Eq. 2), 而使用 L9b 反应可 $100 \%$ 转化, 但对映选择性稍差 $(73 \%$ $e e$ ). 对于底物 $\alpha$-乙酰氨基肉桂酸甲酯 $\mathbf{1}$, 使用配体 $\mathbf{L 9 c}$ 与 $\mathrm{Rh}$ 形成的催化剂, 同样条件下反应 $20 \mathrm{~h}$, 即可得到 $80 \% e e$ 目标产物(Eq. 3). 这些结果引起了人们对单齿磷 配体在不对称催化氢化反应中应用的高度关注.

2004 年, 周其林研究组 ${ }^{[37]}$ 报道了手性单齿螺环亚 膦酸酯配体 L10a $\sim \mathbf{L 1 0 e}$ (图 2)在 $\mathrm{Rh}$ 催化的 $\alpha$-和 $\beta$ -

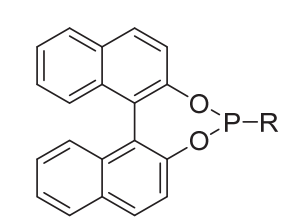

$(S)$-BINOL $(R)$-BINOL $\mathrm{L9b} R=\mathrm{Ph}$ L9c $\mathrm{R}=\mathrm{Me}$

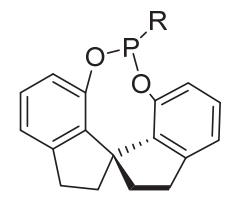

$\begin{array}{ll}\mathrm{L} 10 \mathrm{a} R=\mathrm{C}_{6} \mathrm{H}_{5} & \mathrm{~L} 11 \mathrm{a} \mathrm{R}=\mathrm{CH}_{3} \\ \mathrm{~L} 10 \mathrm{~b} \mathrm{R}=4-\mathrm{MeC}_{6} \mathrm{H}_{4} & \text { L11b R }=i-\mathrm{Pr}\end{array}$

L10c R $=4-\mathrm{MeOC}_{6} \mathrm{H}_{4}$ L11c $\mathrm{R}=t-\mathrm{Bu}$

L10d R $=4-\mathrm{ClC}_{6} \mathrm{H}_{4}$

L10e R $=4-\mathrm{CF}_{3} \mathrm{C}_{6} \mathrm{H}_{4}$

图 2 诱导效果好的的单齿亚膦酸酯配体

Figure 2 Well-induced mono-phosphonites ligands

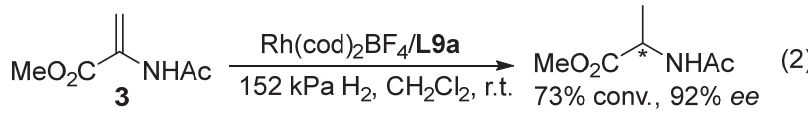

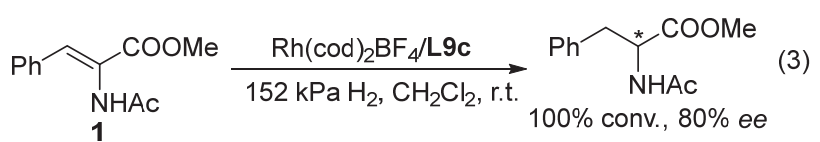

脱氢氨基酸衍生物的不对称氢化反应中的应用. 配体的 电子效应对反应有着重要影响，当 $\mathrm{R}$ 基团的苯基上连接 吸电子取代基时会显著降低催化剂的活性和选择性. 使 用配体 L10c, 无论是 $\alpha$-乙酰氨基肉桂酸甲酯 $\mathbf{1}$ 还是 $\beta$ 乙酰氨基肉桂酸甲酯衍生物 $Z / E$ 混合物 7 , 这类较难被 氢化的底物, 反应均获得最满意的结果, 底物完全转化 且对映选择性分别高达 $99 \% e e$ 和 $98 \% e e$ (Scheme 3).

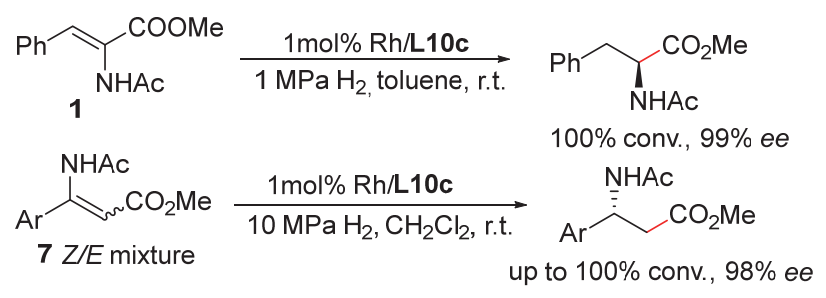

图式 3 L10c 在不对称氢化中的应用

Scheme 3 Application of L10c in AH

$N, N$-二烷基烯胺是一类极具挑战的底物, 不同于烯 酰胺, 它的结构中没有酰胺键这样的极性官能团, 无法 在过渡态形成时与金属配位形成环状螯合物. 2006 年, 周其林等 ${ }^{[38]}$ 将螺环配体 L11a L11c 用于二烷基烯胺 8 的不对称氢化中，提供了直接合成手性叔胺的方法. 在 该体系里，同时添加碘单质和乙酸可以使对应的 $\mathrm{Rh}$ 催 化剂发挥最好的效果. 配体上 $\mathrm{R}$ 基团的大小也影响着反 应的对映选择性, $\mathrm{R}$ 越大，选择性越好，所以取代基为叔 丁基的配体 L11c 手性诱导效果最好(Eq. 4).

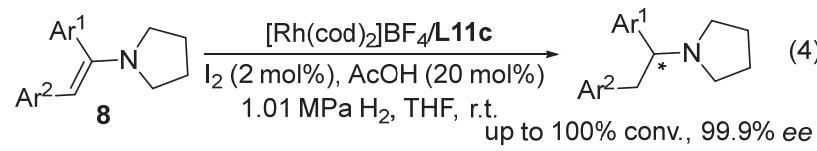


2006 年, Martin 等 ${ }^{[39}$ 报道了一种含有双亚膦酸酯配 体 $\mathrm{L12}$ 和环己二铵配体的 $\mathrm{Ru}$ 配合物, 并将其用于酮 9 的不对称氢化反应中, 反应最高可 $100 \%$ 转化并获得 97\% ee 值(Eq. 5).

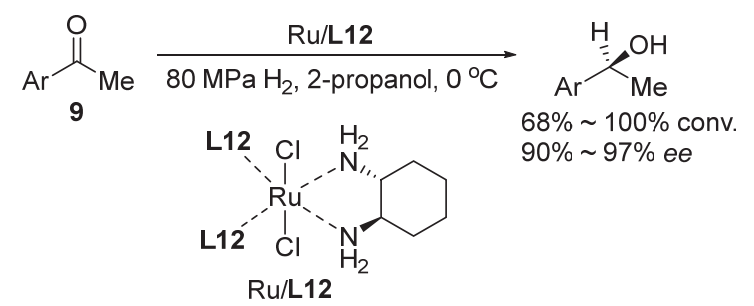

\section{3 单齿亚磷酸酯(Phosphites)配体}

亚磷酸酯的应用是不对称催化领域的一个重大突 破. 与膦配体(phosphine)相比, 亚磷酸酯(phosphites) 只 需由廉价易得的醇经过 1 2 步的反应即可制得, 且对 空气和其他氧化剂稳定. 但这类配体对亲核试剂的稳定 性较差, 也容易发生水解、醇解等副反应, 研究发现, 当 其结构中含有大位阻取代基团时, 可以抑制这些副反应

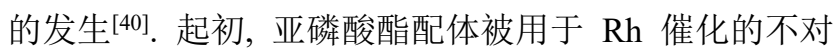
称氢甲酰化反应 ${ }^{[41]}$ 和 $\mathrm{Cu}$ 催化的 1,4-共轭加成反应 ${ }^{[42]}$, 之后陆续被成功用于其他不对称催化反应, 其中最大的 突破是在潜手性底物的不对称氢化中的应用. 下面根据 手性骨架的分类对亚磷酸酯配体依次进行介绍.

\subsection{BINOL 骨架配体}

BINOL 骨架是几种手性骨架中最早大量设计用于 不对称氢化中的，在 2000 2010年期间，对这类配体的 研究呈现泉涌式爆发, 且逐渐趋于成熟.

2000 年, Reetz 课题组 ${ }^{[4]}$ 发现, 基于 BINOL 的亚磷 酸酯配体 L13 和 $\mathrm{L14a} \sim \mathrm{L14c}$ (图 3)在 $\mathrm{Rh}$ 催化的衣康酸 二甲酯 $\mathbf{2}$ 的不对称氢化中表现出媲美双齿磷配体的催化 效果. 反应可完全进行, 以 $96.2 \% \sim 99.6 \% e e$ 得到 $(S)$-构 型的还原产物, 在使用 $\mathbf{L 1 4 b}$ 时, $S / C$ 的比例升至 5000, 反应依旧可以完全转化, 且对映选择性在 $99 \%$ ee 以上. 此外, 他们还发现 $\mathrm{Rh}$ 与配体的比例对反应的对映选择 性几乎没有影响. L14a L14c 在底物 $\alpha$-乙酰氨基丙烯 酸甲酯 3 的不对称氢化中诱导能力也极强, 产物可获得 $90 \%$ 以上 $e e$ 值. 配体 $\mathbf{L 1 4}$ 的合成及在不对称催化氢化中 的成功应用打破了不对称催化领域内双齿配体优于单 齿配体的固有观念.

Reetz 的报道奠定了单齿磷配体发展的基石, 在此 基础上, 各种对 BINOL 骨架进行结构修饰的亚磷酸酯 配体被陆续报道, 利用亚磷酸酷配体的易修饰性探索不 同底物。

所适用的配体, 主要包括以下三个方向: 一是改变 配体中的烷氧基部分, 得到配体 $\mathbf{L 1 4 d} \sim \mathbf{L 1 4 x}$, 将它们
用于 $\mathrm{Rh}$ 催化的 $\alpha$-乙酰氨基肉桂酸甲酯 1 、衣康酸二甲 酯 2、 $\alpha$-乙酰氨基丙烯酸甲酯 3 、以及烯酰胺的不对称 氢化中, 均取得了不错的催化效果 ${ }^{[43-51]}$, 其中 $\mathbf{L 1 4 m} \sim$ L14n 作为配体用于 $\beta$-乙酰氨基丙烯酸酯 10a $\sim 10 \mathrm{~b}$ 的不 对称氢化时, 氢化产物的构型与联荎骨架的手性是一致 的, (S)-BINOL 对应 $S$ 构型的还原产物, $(R)$-BINOL 相应 得到 $R$ 构型产物 ${ }^{[45]}$.

二是用 $\mathrm{H}_{8}$-BINOL 替换 BINOL 骨架，早在单齿磷配 体兴起前, 基于 $\mathrm{H}_{8}$-BINOL 的膦配体就已成功用于各种 不对称催化反应中[52]. 2003 年, Bakos 等 ${ }^{[53]}$ 将 $\mathbf{L 1 5 b} \sim$ L15c 用于衣康酸二甲酯 2 的不对称氢化中, 含有该配体 的 $\mathrm{Rh}$ 催化剂是有一定优越性的, 当催化剂的转化数 $(S / C)$ 高达 40000 时, 反应的转化率和对映选择性均仍能 保持在 $90 \%$ 以上. 基于碳硼烷和 $\mathrm{H}_{8}$ - $\mathrm{BINOL}$ 的亚磷酸酯 配体 L15a 也被 Lyubimov 小组 ${ }^{[50]}$ 报道.

三是在联荥酚其中一个酚羟基的邻位上引入新取 代基. 2005 年, Reetz等 ${ }^{[54]}$ 合成了一系列 $C_{1}$ 对称的含手性 $\mathrm{P}$ 立体异构中心的 BINOL 单齿磷配体. 将邻位烷基取代 的单齿亚磷酸酷配体 L16a 用于 $\mathrm{Rh}$ 催化的衣康酸二甲 酯 2 和 $\alpha$-乙酰氨基丙烯酸甲酯 3 的不对称氢化反应中, 发现 P-差向异构配体混合物一起发挥作用时效果最佳, 反应可完全转化, 产物的对映选择性分别为 $91.3 \% e e$ 和 $97 \%$ ee.

\section{2 联苯酚 Tropos 配体}

Tropos 型与 Atropos 型是一组相反的概念 ${ }^{[55]}$, 类似 于 BINAP (1, '1'-联萗-2,2'-双二苯膦), 绕轴旋转受阻的联 苯分子称为轴稳定(Atropos)的分子, 即具有稳定的轴手 性; 而能绕轴自由旋转的分子, 则称为轴不稳定 (Tropos)的分子, 此类分子, 由于构型的快速转化, $R$ 构 型和 $S$ 构型这对对映异构体无法分离得到.

可以通过在 Tropos 配体上引入手性基团使配体兼 具诱导源手性和轴手性, 以此来诱导合成出所需的手性 催化剂. 引入手性基团的方法主要有两种, 其一, 使用 手性醇诱导合成( $(\mathbf{1 7} \sim \mathbf{L 1 8}, \mathbf{L 2 1})$, 其二, 可在 Tropos 亚 磷酸酯配体的联苯骨架上引入手性基团(L19)(图 4). 合 成的配体同时包含诱导源手性和联苯轴手性, 会形成非 对映异构体，由于非对映异构体物化性质不同，可以经 过简单的柱色谱分离, 同时由于能量不同, 诱导轴手性 的过程中产物可能会倾向某种构型, 甚至得到单一构型 产物.

2001 年，肖建良等 ${ }^{[56]}$ 报道了 Tropos 配体 L17 L18. 该配体以等量的非对映异构体混合物的形式存在, 然而 当该混合物与 $\mathrm{Rh}(\mathrm{II})$ 前体反应后, 均势被打破, 形成的 两种非对映异构体的 $\mathrm{Rh}$ 配合物比例为 $1: 5$. 在衣康酸 二甲酯 2 的不对称氢化中, 当引入的手性醇部分为手性 
MeOOC $\sim_{2}$ COOMe

L13, 14a 14c: 100\% conv. $96.2 \% \sim 99.6 \%$ ee

L14m:100\% conv., 95.2\% ee

L140: $100 \%$ conv., $83 \%$ ee

L14r+L14t: 100\% conv., 99\% ee

L14u L14v: 100\% conv., $99.7 \% \sim 99.8 \%$ ee

L14w: $100 \%$ conv., $98 \%$ ee

L15b L15c: $91.79 \% \sim 100 \%$ conv., $96.9 \% \sim 97.6 \%$ ee

L16a: $100 \%$ conv., $91.3 \%$ ee

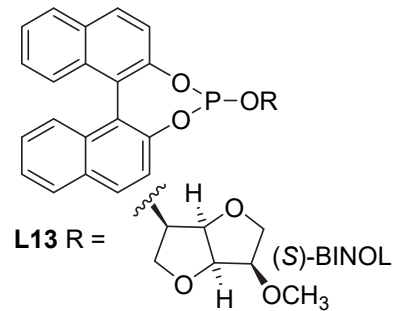

L14a R $=i-\operatorname{Pr}(S)-\mathrm{BINOL}$

L14b $\mathrm{R}=(R)$-1-phenylethyl $(S)$-BINOL

L14c R $=(S)$-1-phenylethyl ( $(S)$-BINOL

L14d $\mathrm{R}=c-\mathrm{C}_{5} \mathrm{H}_{9}(S)-\mathrm{BINOL}$

L14e R $=\mathrm{CH}_{2} \mathrm{Ph}(S)$-BINOL

L14f $\mathrm{R}=\mathrm{CH}_{2} \mathrm{CH}\left(\mathrm{CH}_{3}\right)_{2}(R)-\mathrm{BINOL}$

L14g R $=\mathrm{CH}_{2} \mathrm{CH}\left(\mathrm{C}_{2} \mathrm{H}_{5}\right)_{2}(R)$-BINOL

L14h R $=\mathrm{CH}_{2} \mathrm{C}\left(\mathrm{CH}_{3}\right)_{3}(\mathrm{~S})-\mathrm{BINOL}$

L14i R $=(\mathrm{S})-\mathrm{CH}\left(\mathrm{CH}_{3}\right)\left(\mathrm{C}_{2} \mathrm{H}_{5}\right)(\mathrm{S})-\mathrm{BINOL}$

L14j $\mathrm{R}=(\mathrm{S})-\mathrm{CH}\left(\mathrm{CH}_{3}\right)\left(\mathrm{C}_{2} \mathrm{H}_{5}\right)(R)-\mathrm{BINOL}$

L14k R $=(R)-\mathrm{CH}(\mathrm{Ph})\left(\mathrm{CH}_{3}\right)(\mathrm{S})-\mathrm{BINOL}$

L14I $\mathrm{R}=\mathrm{CH}_{2} \mathrm{CH}_{2} \mathrm{Cl}(R)-\mathrm{BINOL}$

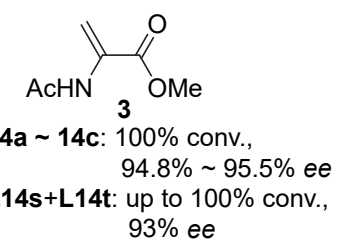

L14n: up to $100 \%$ conv, $85 \%$

L16a: $100 \%$ conv., $97 \%$ ee
$\mathrm{R}^{1} \overbrace{\mathrm{m}}^{\mathrm{NHAc}} \mathrm{COOR}^{2}$

10a $R^{1}=M e, R^{2}=M e$

10b $R^{1}=M e, R^{2}=E t$

L14m L14n:

$$
\mathrm{R}^{1 \uparrow} \mathrm{RHAc}^{2}
$$

$4 \mathrm{R}^{1}=\mathrm{H}, \mathrm{R}^{2}=\mathrm{Ph}$

11a $R^{1}=H, R^{2}=2-N a p$ L14p: $100 \%$ conv.,

11b $\mathrm{R}^{1}=\mathrm{CH}_{3}, \mathrm{R}^{2}=\mathrm{Ph}$

up to $100 \%$ conv., $94 \%$ ee $11 c \mathrm{R}^{1}=\mathrm{H}, \mathrm{R}^{2}=p-\mathrm{ClC}_{6} \mathrm{H}_{4}$

L14d 14I: > 99.5\% conv.

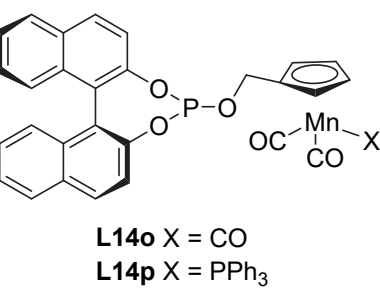

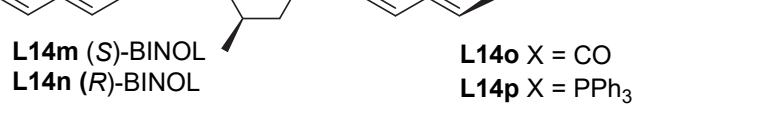<smiles>[R]c1cc(COp2oc3ccc4ccccc4c3c3c(ccc4ccccc43)o2)cc([R])c1[R]</smiles>

L14r $\mathrm{R}^{1}=\mathrm{OMe}, \mathrm{R}^{2}=\mathrm{H}, \mathrm{R}^{3}=\mathrm{OMe}$

L14s $R^{1}=H, R^{2}=$ OMe, $R^{3}=$ OMe<smiles>CC(C)(C)OPOc1ccc2ccccc2c1-c1c(OCc2c(F)c(F)c(F)c(F)c2F)ccc2ccccc12</smiles><smiles></smiles>
$\mathrm{R}=-\mathrm{L}$

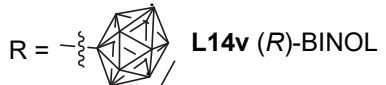

$R=-$ L14w (S)-BINOL

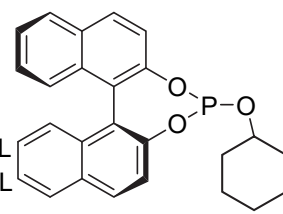

L14x

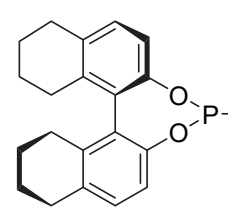

L15a

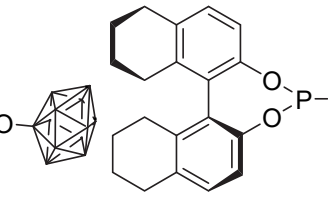

L15b R $=\mathrm{CH}\left(\mathrm{CH}_{3}\right) \mathrm{Ph}$

L15c R $=i-\mathrm{Pr}$

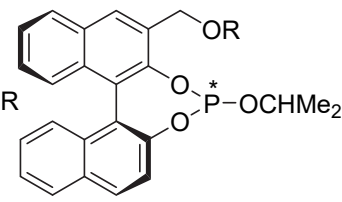

L16a R $=\mathrm{CH}_{2} \mathrm{Ph}$

图 3 BINOL 骨架的单齿亚磷酸酯配体及其诱导不对称氢化的底物

Figure 3 A summary of phosphites ligands based on BINOL and their counterpart substrates in $\mathrm{AH}$

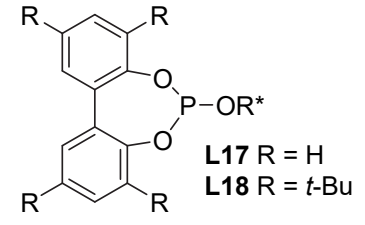<smiles>CCOc1cccc2op(OC(C)C)oc3cccc(OPOC(C)C)c3c12</smiles>

L19<smiles></smiles><smiles>COp1oc2ccccc2c2ccccc2o1</smiles>

L20

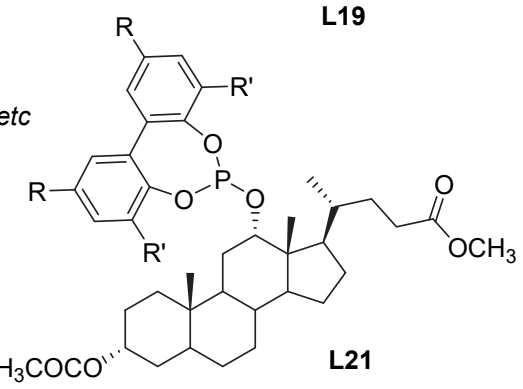

图 4 Tropos 亚磷酸酯配体

Figure 4 Tropos phosphite ligands

樟脑醇时, 配体 $\mathbf{L 1 7}$ 对应的 $\mathrm{Rh}$ 络合物催化效果最好, 反应获得了 $100 \%$ 转化率和 $75 \%$ ee 的对映选择性.

2003 年, Rampf 等 ${ }^{[57]}$ 合成 Tropos 配体 L19, 在 Rh 催化的不对称氢化中, 分别以 $100 \%$ 转化率、 $96 \% \mathrm{ee}$ 和 93\% ee 得到衣康酸二甲酯 $\mathbf{2}$ 和 $\alpha$-乙酰氨基丙烯酸甲酯 3 的氢化产物, 但该配体在 $\beta$-脱氢氨基酸衍生物和 $\alpha$-芳基
烯酰胺衍生物的不对称氢化中的适用性较差, 尽管转化 率尚可, 但对映选择性在 $8 \% \sim 81 \%$ ee 不等.

2007 年, Iuliano 小组 ${ }^{[58]}$ 合成了一种由乙酰脱氧胆酸 甲酯诱导出轴手性的 Tropos 联苯亚磷酸酯配体 L21, 并 将其应用于衣康酸二甲酯 2 的不对称氢化反应中, 在 $700 \mathrm{kPa}$ 氢气压力下, 室温下反应 $20 \mathrm{~h}$, 原料可以完全转 化，对映选择性最高可达 $91 \%$ ee.

Tropos 磷配体在组合化学中的应用也取得了一些 成果. 2005 年, Reetz 等 ${ }^{[59]}$ 报道了手性亚磷(膦)酸酯与 Tropos 亚磷酸酯的异种组合在催化氢化 $\beta$-乙酰氨基丙 烯酸酯中的应用. 他们发现 L9d 与 L20 的异种组合达到 的催化效果最优，最高可获得 $100 \%$ 转化率和 $98 \%$ ee (Eq. 6). $\mathbf{L 2 0}$ 作为 Tropos 配体, 其绝对构型在 $R 、 S$ 之间 可以快速转化(Eq. 7), 所以与 L9d 形成的异种配合物以 非对映异构体的形式在溶液中存在(Eq. 8). 进一步分离 研究发现, 异种组合催化剂构型为 $(R, R)$ 时, 产物的 $e e$ 值 可达到 $99 \%$, 而 $(R, S)$ 对应只有 $40 \%$, 说明构型的协同作 用有利于不对称诱导.

\section{3 糖基配体}

为了解决普通单齿亚磷酸酯配体 $\mathrm{M}-\mathrm{P}$ 键自由旋转 


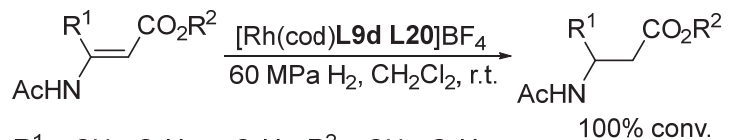

$\mathrm{R}^{1}=\mathrm{CH}_{3}, \mathrm{C}_{2} \mathrm{H}_{5}, n-\mathrm{C}_{3} \mathrm{H}_{7} ; \mathrm{R}^{2}=\mathrm{CH}_{3}, \mathrm{C}_{2} \mathrm{H}_{5} \quad \begin{aligned} & 100 \% \text { conv. } \\ & 95 \% \sim 98 \% \text { ee }\end{aligned}$<smiles>COp1oc2ccccc2c2ccccc2o1</smiles><smiles></smiles><smiles>COp1oc2ccccc2c2ccccc2o1</smiles><smiles>COP(OC)Oc1ccccc1-c1ccccc1O</smiles>

$\left[\mathrm{Rh}(\operatorname{cod}) \mathrm{L9d}(R)-\mathrm{L20}_{\mathrm{B} F} \rightleftharpoons\left[\mathrm{Rh}(\operatorname{cod}) \mathbf{L} \mathbf{d} \mathrm{d}(S)-\mathrm{L20}_{4}\right] \mathrm{BF}_{4}\right.$

引起的在某些不对称催化反应中手性诱导能力低的问 题, Reetz 课题组 ${ }^{[60]}$ 和郑卓等 ${ }^{[61-63]}$ 基于糖基发展了一系 列新的单齿亚磷酸酯配体(图 5), 这类配体的糖基上富 含氧原子, 可与金属进行半不稳定配位, 从而有效抑制 $\mathrm{M}-\mathrm{P}$ 键的旋转, 减少了过渡态旋转异构体的数目, 有 利于系统实现高对映选择性.
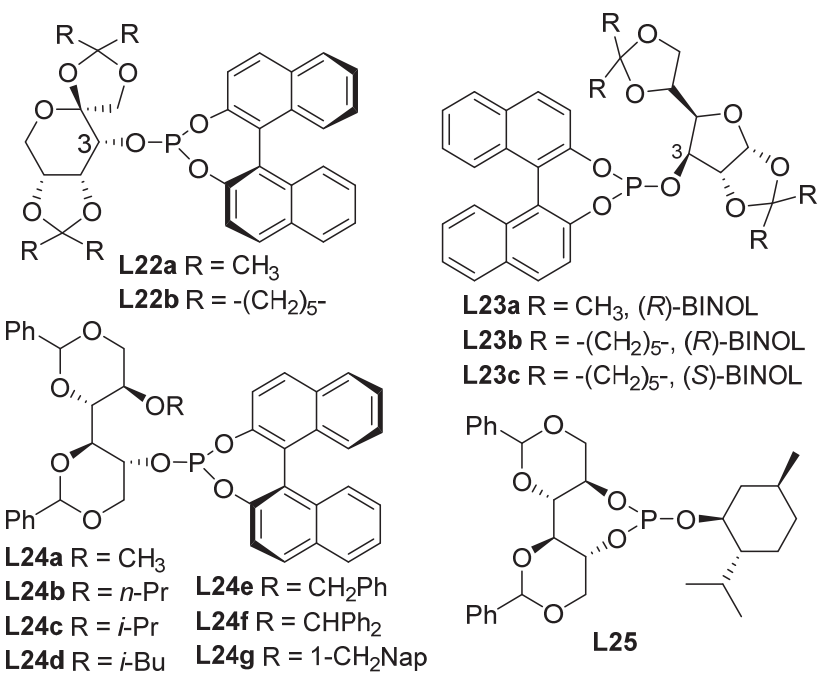

L23a $\mathrm{R}=\mathrm{CH}_{3},(R)-\mathrm{BINOL}$ L23b R $=-\left(\mathrm{CH}_{2}\right)_{5^{-}},(\mathrm{R})-\mathrm{BINOL}$ L23c R $=-\left(\mathrm{CH}_{2}\right)_{5},,(\mathrm{~S})-\mathrm{BINOL}$<smiles>CC1CCC(OP(OC2COC(c3ccccc3)OC2C)OC2COC(c3ccccc3)OC2C(C)C)CC1</smiles>
L25

图 5 优异的单齿糖基亚磷酸酯配体

Figure 5 Excellent glycosyl mono-phosphite ligands

Reetz等 ${ }^{600}$ 将 $D$-葡萄糖衍生的单齿亚磷酸酯配体用 于 $\mathrm{Rh}$ 催化的烷基烯醇酯 12 的碳碳双键不对称还原氢化 中, 这类底物因结构中缺少可与金属中心配位的原子导 致手性诱导效应差，即使用双齿膦配体效果也不理想， 但糖基配体 L23a 的使用却收获了令人满意的效果, 反 应完全转化，产物对映选择性高达 $94 \% e e$ (Eq. 9).

郑卓等 ${ }^{61]}$ 将 $D$-果糖和 $D$-葡萄糖衍生的单齿亚磷酸 酯配体用在 $\alpha$-芳基烯酰胺和衣康酸二甲酯 2 的不对称氢 化反应中, 两种底物的氢化难易程度差异较大, 多数配

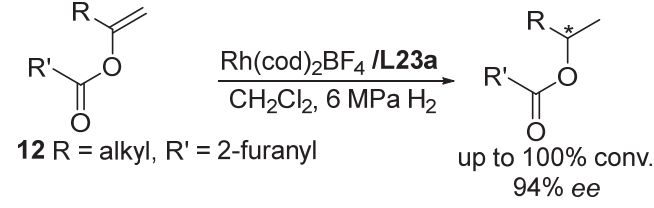

体在衣康酸二甲酯的不对称氢化中表现优异，而烯酰胺 适用的配体相对较少，其中，配体 L22a $\sim \mathbf{L 2 2 b}$ 和 L23a L 23c 在两种底物的氢化中均表现良好, 获得了 高于 $87.7 \%$ ee 的对映选择性. 此外, 他们还发现了糖基 骨架上 C-3 的构型对产物的对映选择性有着重要影响, $D$-果糖配体的 C-3 为 $R$ 构型时诱导能力比 $S$ 构型强, $D$ 葡萄糖配体则与之相反. BINOL 骨架的构型也影响对映 选择性，当 BINOL 骨架的构型与 C-3 构型一致时，产物 的对映选择性最优. 次年, 他们将相同系列配体用于 $\alpha-$ 脱氢氨基酸酯和 $(Z)-\beta$-脱氢氨基酸酯的不对称氢化 中[62a]，但仅 $\mathbf{L 2 3 b}$ 表现优异. 同年，他们报道了甘露醇 衍生的单齿亚磷酸酯配体 L 24a L 24g 在衣康酸二甲酯 2 、烯酰胺衍生物、 $\alpha$-脱氢氨基酸酯和 $\beta$-脱氢氨基酸酯 等不对称氢化中的应用, 反应的对映选择性在 $94.4 \% e e$ 以上 ${ }^{[62 b]}$. 而且在前两种底物的不对称氢化中, 催化剂 用量更是低至 $0.02 \mathrm{~mol} \%$, 反应转化率和对映选择性也 只有轻微的下降.

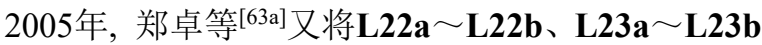

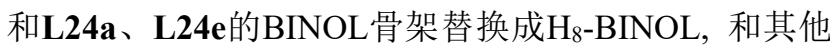
类型的 $\mathrm{H}_{8}-\mathrm{BINOL}$ 配体相比, 这类配体在不对称氢化反 应中更具优越性，在 $\alpha$-芳基烯酰胺衍生物和衣康酸二甲 酯 2 的不对称氢化中反应均可以获得 $95 \% \mathrm{ee}$ 以上对映选 择性. 次年, 他们发现配体 $\mathbf{L 2 4 g}$ 在 $\beta$-邻苯二甲酰亚胺丙 烯酸酯 13 的不对称氢化中表现优异 ${ }^{[63 b]}$, 可用于合成手 性 $\beta^{2}$ 氨基酸. 反应最高可完全转化，并获得 $99.1 \% e e$ 的 对映选择性(Eq. 10).

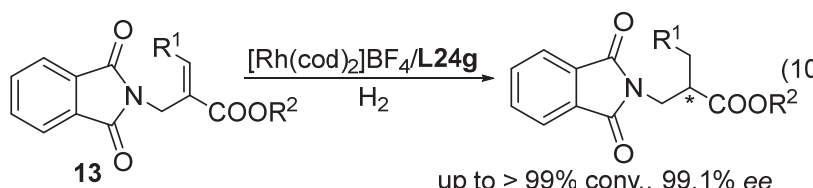

通过在 $\mathbf{L} 23$ 呋喃基的 C-3 位上引入不同的取代基, Elisabetta 等 ${ }^{[64]}$ 开发了第二代 $D$-葡萄糖配体，他们发现 在 C-3 位引入取代基有利于衣康酸二甲酯 2 和脱氢氨基 酸衍生物的不对称氢化 $(99.9 \% e e, 85 \% e e)$, 但不利于烯 酰胺的不对氢化 $(53 \% \sim 67 \% e e)$. 说明取代基对 $e e$ 值的 影响不仅由呋喃糖苷 C-3 位构型和 BINOL 的构型决定, 也和底物有关. 2017 年, Assem 等 ${ }^{[65]}$ 将 $D$-甘露糖衍生的 单齿亚磷酸酯配体 $\mathbf{L 2 5}$ 用于 $\mathrm{Ru}$ 催化 $\alpha, \alpha, \alpha$ 三氟苯乙酮 的不对称催化氢化, 反应可完全转化, 分离收率为 $83 \%$, ee 值达至 $73 \%$. 


\section{$3.4 C_{3}$ 对称的单齿配体}

2009 年, Reetz 等 ${ }^{[6]}$ 报道了螺旋结构 $C_{3}$ 对称的单齿 亚磷酸酯配体, 它含有一个螺旋三骨架结构, 当 $\mathrm{R}$ 基团 与 $\mathrm{P}$ 原子在三个氧原子构成的平面的同侧时, 称为 “syn”，反之则为 “anti” (图 6a). 整个配体分子具有 左、右手两种不同的螺旋结构, 用 “P、M” 表示(图 6b). 通过在 $C_{3}$ 对称的手性配体 $\mathrm{P}(\mathrm{OR})_{3}$ 上引入大位阻基团金 刚烷羧酸诱导出螺旋手性, 进而将螺旋结构锁定在 $\mathrm{P}$ 或 $M$ 构型，使得形成的非对映螺旋异构体之间不会快速互 相转化. 将配体 L26a 用于 $\mathrm{Rh}$ 催化的难氢化底物 2-芳基 烯丁醇 14 的不对称氢化中, 反应取得了优异的结果, 底 物几乎完全转化, 对映选择性高达 $98 \%$ ee (Eq. 11). 他 们还发现无论是 $(R, R, R)$-L26a 还是 $(S, S, S)$ - L26a 均为 “syn” 构象, 且由于 BINOL 骨架的绝对构型不同, 导 致前者螺旋手性锁定为 $\mathrm{P}$ 构型, 后者为 $\mathrm{M}$ 构型; 此外, 空间位阻较小的配体 L26b 会呈现出更多的空间构型, 即同时存在 $\mathrm{P}$ 和 $\mathrm{M}$ 构型, 所以产生的对映选择性极低 $(32 \% e e)$.

a
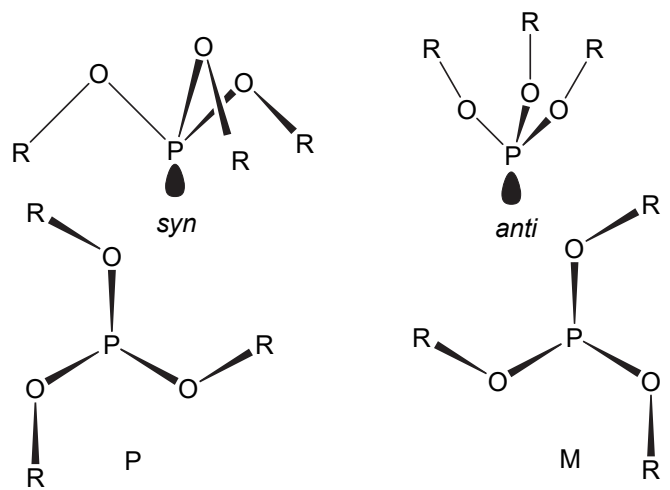

图 6 (a) $C_{3}$ 对称的亚磷酸酯配体的顺反异构方式和(b) $C_{3}$ 对 称的亚磷酸酯配体的螺旋异构方式

Figure 6 (a) Syn- and anti-conformeric forms of $C_{3}$-symmetric phophites; (b) $\mathrm{P}$ and $\mathrm{M}$ helical enantiomers of $C_{3}$-symmetric phosphites
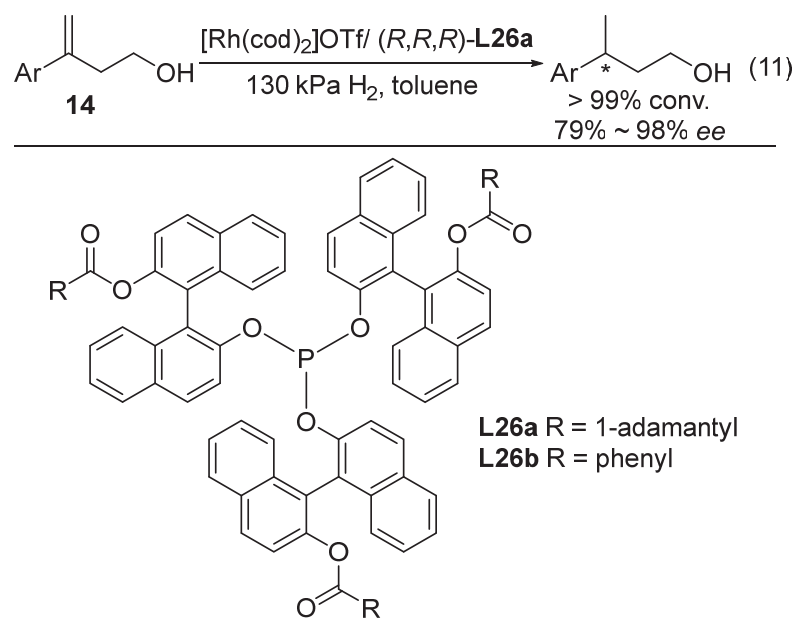

2014 年, Pereira 等 ${ }^{[67]}$ 将 L26a 用于 $\mathrm{Rh}$ 催化的衣康酸 二甲酯 2 的不对称氢化中，但诱导效果不好，仅 $62 \% e e$, 反应也没有完全转化, 可见这类配体的底物适用范围并 不广.

\section{5 螺环骨架配体}

2012年，周其林等[68]将螺环配体用于 $\beta$-芳基烯酰胺 的不对称氢化中，解决了 $(E)-\beta$-芳基烯酰胺选择性差的 问题. 螺环配体 $(S)-\mathbf{L} 27 \mathbf{a}$ 的使用可使反应以 $100 \%$ 转化 率、 $91 \% \sim 95 \%$ ee 获得 $(Z)-\beta$-芳基烯酰胺 15a 的还原产物, 而 $(R)-\mathbf{L} 27 \mathbf{b}$ 在 $(E)-\beta$-芳基烯酰胺 $\mathbf{1 5 b}$ 中表现非常优异 (100\%转化率、 $81 \% \sim 97 \% e e)$ (Eq. 12). 该氢化反应是获 取具有光学活性的 $\beta$-芳基异丙胺一一一类常见的生物 活性中间体的有效方法，具有重要意义.

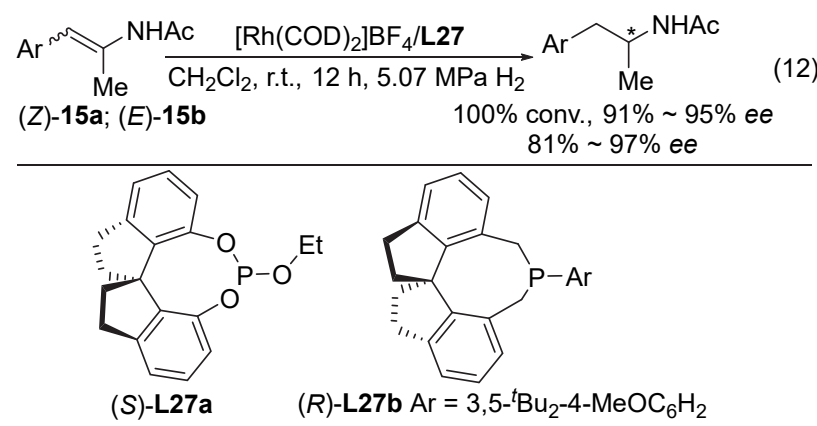

\section{4 单齿亚磷酰胺(Phosphoramidites)配体}

单齿亚磷酰胺配体是在亚磷酸酯配体的基础上将 其中的一个磷-氧键换成磷-氮键, 相比较亚膦酸酯和亚 磷酸酯，亚磷酰胺配体显示了更好的稳定性和更优异的 配位能力 ${ }^{[69]}$, 被称作是 “优势手性配体” [70]. 下面根据 亚磷酰胺配体的手性骨架进行分类，对近年来亚磷酰胺 配体在不对称氢化中取得的进展进行介绍.

\subsection{BINOL 配体}

以 BINOL 为手性骨架的亚磷酰胺配体同前两类单 齿磷配体一样，应用非常广泛. 1994 年, Feringa 等[71]报 道了单齿亚磷酰胺配体 L28a (MonoPhos)的合成(图 7), 后被用于 $\mathrm{Cu}$ 催化的不对称 1,4-加成反应中[72]. 2000 年, Feringa 等 ${ }^{[5}$ 将该配体应用于不对称催化氢化中, 底物 $\alpha$ 乙酰氨基肉桂酸甲酯 1 完全转化, 产物对映选择性达到 95\% ee. 而且该配体具有良好的底物适用性，在其他 $\alpha$ 脱氢氨基酸衍生物、衣康酸衍生物和 $\alpha$-芳基烯酰胺的不 对称氢化中都具有优秀的立体选择性 ${ }^{[5,73-74]}$. MonoPhos 适用的氢气压力范围比双齿配体更广, 无论在高压还是 低压氢气中, 诱导能力几乎不变且反应速率随压力的升 高而加快; 但是该配体有溶剂依耐性，在非质子溶剂如 二氯甲烷、乙酸乙酯中的诱导活性比在质子溶剂中要好 一些. MonoPhos 的出现以及在不对称催化氢化中的成 

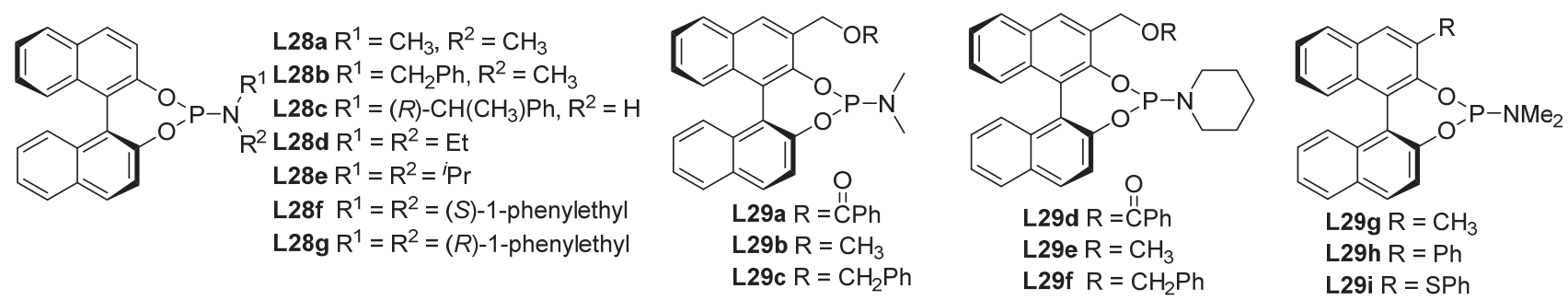

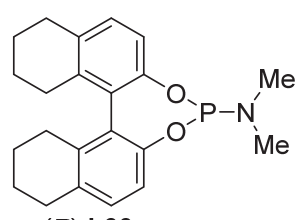

(R)-L30a

(S)-L30b

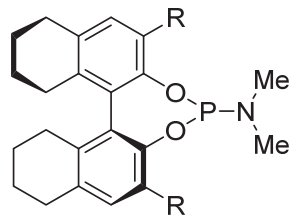

L30c R = 2,4,6- $\left(\mathrm{CH}_{3}\right)_{3} \mathrm{C}_{6} \mathrm{H}_{2}$ L30d R = 4- $\mathrm{CH}_{3} \mathrm{O}-\mathrm{C}_{6} \mathrm{H}_{4}$

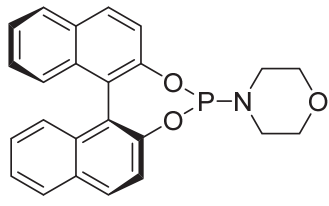

L31a

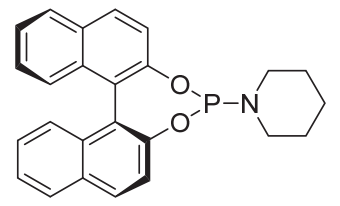

L31b

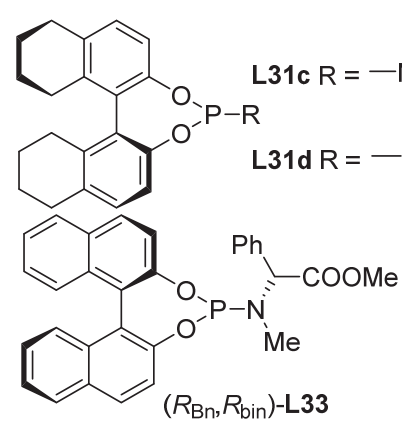<smiles>[R]P(OPc1ccc2ccccc2c1-c1c(OP)ccc2ccccc12)[IH]C</smiles>

L31e $\mathrm{R}=\mathrm{NHCH}\left(\mathrm{CH}_{3}\right)_{2}$ L31f R = NH( $\left(\mathrm{CH}_{2}\right)_{3} \mathrm{CH}_{3}$ L31g R = NHC $\left(\mathrm{CH}_{3}\right)_{3}$

Figure 7 Phosphoramidite ligands derived from BINOL and $\mathrm{H}_{8}-\mathrm{BINOL}$

功应用迅速得到了学术界和工业界的高度重视, 该类配 体及催化剂的专利已授权给DSM公司. 当然, MonoPhos 的适用范围也是有限的, Feringa等 ${ }^{[75]}$ 尝试将它用于 $\beta$-脱 氢氨基酸酯的不对称氢化中，但结果并不理想，反而是 $\mathbf{L 2 8 b} \sim \mathbf{L 2 8 c}$ 表现优异，产物的 $e e$ 值在 $92 \% \sim 99 \%$ 范围 内, 且多数情况下反应可以完全转化.

次年，陈新滋等 ${ }^{[76]}$ 合成了 Et-MonoPhos，即 L28d, 其在 $\alpha$ 芳基烯酰胺和 $\beta$ 位芳基取代的 $\alpha$-脱氢氨基酸酯的 不对称氢化反应中的表现优于MonoPhos, 对映选择性 达到 $93 \% \sim 99.6 \% e e$ ，且对应的产率一般在 $99 \%$ 以上.

2005 年, Reetz 等 ${ }^{[54]}$ 合成了含 $\mathrm{P}$ 立体异构中心的 BINOL 邻位取代的配体 $\mathbf{L 2 9} \mathbf{a} \sim \mathbf{L 2 9}$, 在 Rh 催化的标准 底物 2 和 3 的不对称氢化反应中, 使用 L29a $\sim$ L29f, 反 应均完全转化, 对映选择性最高分别达 $96.8 \% e e$ 和 $99 \%$ $e e$, 且 $\left(S_{\text {binol }}, S_{\mathrm{P}}\right)-\mathbf{L} 29 \mathbf{a} \sim\left(S_{\text {binol }}, S_{\mathrm{P}}\right)-\mathbf{L 2 9 f}$ 引起的对映选择 性比 $\left(S_{\text {binol }}, R_{\mathrm{P}}\right)-\mathbf{L} 29 \mathbf{a} \sim\left(S_{\text {binol }}, S_{\mathrm{P}}\right)-\mathbf{L 2 9 f}$ 高; 而使用 $\mathbf{L 2 9} \sim$ L29i, 发现配体为 $\left(S_{\text {binol, }} S_{\mathrm{P}}\right)$ 或 $\left(S_{\text {binol }}, R_{\mathrm{P}}\right)$ 构型时不对称诱 导能力均较低, 但非对映异构混合物一起发挥催化作用 时, 产物的对映选择性高达 $98 \%$ ee . 虽然两类配体在发 挥作用时存在一些差异, 但是手性 BINOL 骨架始终在 不对称氢化中占主导地位, 而手性磷原子的引入只起辅 助性作用.
2002 年，蒋耀忠等 ${ }^{[77-78]}$ 合成了 $\mathrm{H}_{8}$-MonoPhos $(R)$ L30a, 解决了 BINOL骨架配体在一些溶剂中的低溶解 度造成的溶剂依耐问题，该配体在 $\mathrm{Rh}$ 催化的 $\alpha$-脱氢氨基 酸酯的不对称氢化中产生了高达 $99.9 \% e e$ 的对映选择 性. 次年, Feringa ${ }^{[73]}$ 合成了相反构型的 $\mathbf{L 3 0 b}$, 其在各类 标准底物的不对称氢化中同样表现优异.

2008 年, Beller 等 ${ }^{[79]}$ 报道了 $\mathrm{H}_{8}-\mathrm{BINOL}$ 骨架亚磷酰 胺配体在 Ir 催化的 $\alpha$-脱氢氨基酸、 $\beta$-脱氢氨基酸 ${ }^{[80]}$ 以及 未官能团化烯酰胺 ${ }^{[81]}$ 三类底物的不对称氢化反应中的 应用，他们发现在 $\mathrm{H}_{8}$-BINOL 骨架 3,3'位引入取代基可 以显著提高配体的诱导活性. 在 $\alpha$-脱氢氨基酸衍生物的 不对称氢化中，使用 $\mathbf{L B O c}$ ，相应的还原产物获得的对映 体过量高达 $98 \%$, 且转化率均在 $99 \%$ 以上. 在 $\beta$-脱氢氨 基酸衍生物的不对称氢化中, $\mathbf{L 3 0 d}$ 效果最好, 且 $E$ 型底 物的结果比 $Z$ 型底物好, 最高获得了 $94 \%$ ee 的对映选择 性. 而在催化烯酰胺 4 时(图 8), 配体 L30d 也有满意的 表现，特别在添加了 $\mathrm{NaClO}_{4}$ 后，反应获得了高于 $99 \%$ 的转化率和 $93 \%$ ee 值. 但是该催化体系适用的底物范 围有限，在其他 $\alpha$-芳基烯酰胺中表现一般(29\% $88 \%$ ee).

2005年, Feringa等[82]发现了(S)-MorfPhos配体L31a 和(S)-PipPhos配体L31b, 这两个配体的底物适用性非常 
广泛, 尤其是PipPhos可以称得上是万能配体. 将它们用 于 $\mathrm{Rh}$ 催化的标准底物 $1 \sim 3$ 的不对称氢化中, 反应均完 全转化且产物的对映选择性 $>98 \% e e$; 在一些烯酰胺的 不对称氢化中同样表现优异，如烯酰胺 $4 、 16 a \sim 16 f$, 反 应大多完全转化且对映选择性也在 $95 \%$ ee以上. 对于 $N-$ 甲酰基脱氢氨基酸酯 $17 \mathrm{a} \sim 17 \mathrm{e}^{[83]}$ 和烯醇酯 $18 \mathrm{a} \sim 18 \mathbf{h}^{[84]}$, 尤其后者较难催化, (S)-PipPhos也取得了不错的效果, 反应都可以完全或接近完全转化, 产物的对映体过量在 $94 \% \sim 99 \%$ 之间.
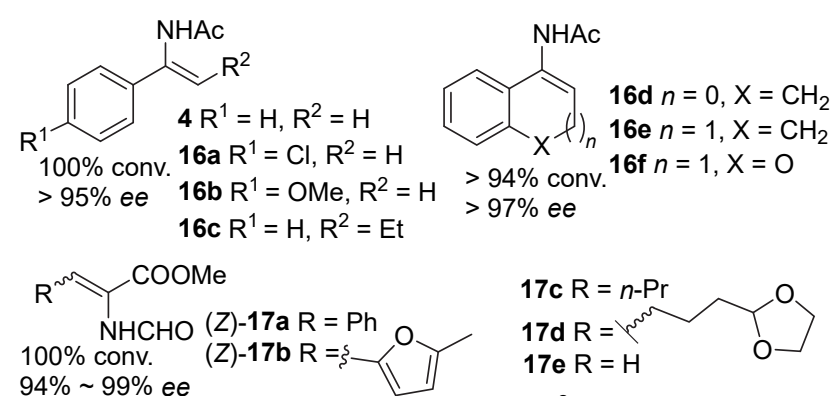
$94 \% \sim 99 \%$ ee
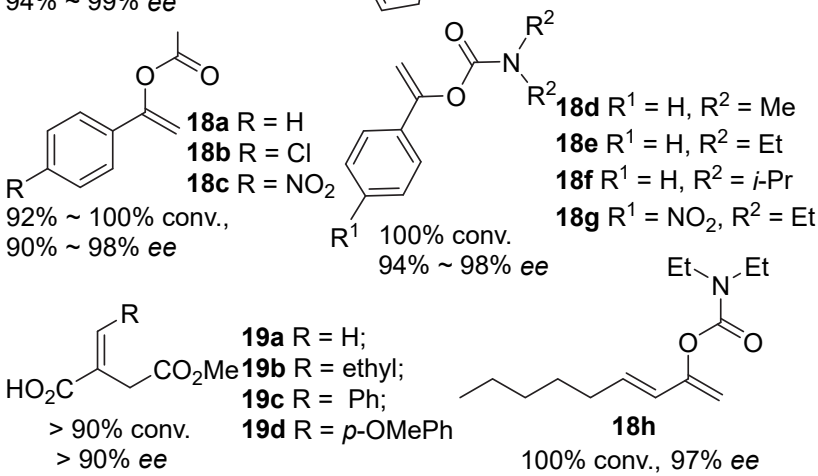

图 $8 \mathrm{Rh} / \mathrm{PipPhos}$ 催化效果好的底物

Figure 8 Substrates catalysted by $\mathrm{Rh} / \mathrm{PipPhos}$ with excellent $e e$

2007 年, de Vries ${ }^{[85]}$ 将 $\mathbf{L 3 1 b} \sim \mathbf{L 3 1}$ 用于 $\mathrm{Rh}$ 催化的 $\beta$-取代的衣康酸单酯 $19 \mathrm{a} \sim 19 \mathrm{~b}$ 的不对称氢化中. 但芳 基取代的底物 19c 19d 催化氢化结果不理想, 而烷基 取代的底物 19a 19b 则取得了良好的氢化结果, 不对 称诱导高达 99.7\% ee, 对于底物 19c 19d, 使用配体 L31d L 31g, 反应的转化率和 $e e$ 值均大于 $90 \%$. 不同 于衣康酸二甲酯或衣康酸, 衣康酸单酯氢化所得产物的 分子末端两个部分可以识别区分, 具有一定的合成价 值.

2008～2009年间，Feringa等 ${ }^{[87]}$ 又将 $(S)$-PipPhos应用 于 Ir催化的一些亚胺和烯胺的不对称氢化中, 包括喹啉 衍生物 $\mathbf{2 0}^{[86]}$ 、喹喔啉衍生物 21 、吲哚衍生物 $\mathbf{2 2}^{[88]}$ 以及 $N$-芳基亚胺 $\mathbf{2 3}^{\left[{ }^{[89}\right.}$ (图9). 在催化氢化底物 20 时, 需要三邻 甲苯基膦作为共配体, 哌啶盐酸盐作为添加剂, 底物可 完全转化, 对映选择性达到 $89 \%$ ee. 而对于喹喔啉衍生 物 21 则无需三邻甲苯基膦作为共配体, 反应仍完全转 化, ee值可达到 $96 \%{ }^{[88]}$. (S)-PipPhos的使用实现了低压下
$N$-芳基亚胺 23 不对称氢化 ${ }^{[89]}$. 底物中与 $\mathrm{N}$ 原子相连的芳 环Ar的电子效应对反应也有影响，当将底物替换为苯环 上2位甲氧基取代的亚胺时，反应可以获得 $97 \% \mathrm{ee}$ ，且 该底物既不易水解，其氢化所得的产物 $\mathrm{N}$ 上的芳基也可 由 TCCA脱除，从而顺利实现伯胺的合成.<smiles>[R]c1ccc2ncccc2c1</smiles>
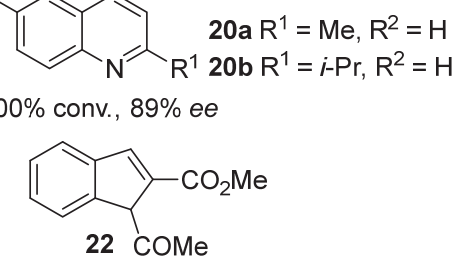

$100 \%$ conv., $74 \%$ ee

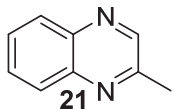

$100 \%$ conv., $96 \%$ ee<smiles>C/C(=N\[Al])c1ccccc1</smiles>

$100 \%$ conv., $97 \% \sim 99 \%$ ee

图 $9 \mathrm{Ir} / \mathrm{PipPhos}$ 诱导效果好的底物

Figure 9 Substrates with excellent $e e$ induced by $\mathrm{Ir} / \mathrm{PipPhos}$

PipPhos 和 MorfPhos 的成功应用激发了配体设计的 新思路. 2007 年, Armspach 课题组 ${ }^{[90]}$ 设计合成了 L32a L32d, 该类配体含两个联䒺手性轴, 将其应用于各种 $\beta$ 芳基取代的 $\alpha$-脱氢氨基酸酯的催化氢化中，反应完全转 化，最终产物 ee 值在 79\% 99\%不等，其中联菜基构型 相反的配体 L32a $\sim \mathbf{L 3 2 b}$ 的手性诱导能力 $(>96 \%$ ee $)$ 比 构型相同的配体 L32c L32d (79\% 86\% ee) 更好，甚 至优于 MorfPhos 和 MonoPhos. 随后，他们对配体结构 进一步改良，将氨基上的联䒺酚手性骨架换成手性氨基 酸骨架, 并应用在相同底物的不对称氢化中, $\left(R_{\mathrm{Bn}}, R_{\mathrm{bin}}\right)-$ $\mathbf{L 3 3}$ 取得了最好的结果，最高获得了 $99 \%$ 转化率和 $92 \%$ $e e$ 的对映选择性 ${ }^{[91]}$. 对于这两类配体而言，尽管其他手 性元素在不对称诱导过程中有一定的影响, 但含磷 BINOL 手性骨架仍起着支配性的关键作用.

2011 年, Lyubimov 等[92]考察了碳硼烷取代的亚磷 酰胺配体 $\mathbf{L 3 4}$ 在 $\alpha$ 和 $\beta$ 脱氢氨基酸衍生物的不对称氢化 反应中的表现. 反应受溶剂影响较大，将溶剂异丙醇替 换成酸性六氟异丙醇, 底物 $\beta$-脱氢氨基酸衍生物对应的 对映选择性可由 $5 \%$ ee 提高至 $85 \%$ ee

2015 年, Leitner 等 ${ }^{[93}$ 探索了亚磷酰胺配体上多手性 元素的催化潜力, 他们从蒎烯衍生物出发合成了配体 L35a L 35b. 在 Rh 催化的衣康酸二甲酯 2 的氢化反应 中 L35a 效果最好，反应几乎完全转化，获得 $95 \%$ ee, 值 得一提的是，当催化剂用量降低至 $0.01 \mathrm{~mol} \%$, 反应的 转化率和对映选择性依旧在 $90 \%$ 以上. 而在 Ir 催化的各 种 $N$-芳基亚胺的不对称氢化中, $\mathbf{L 3 5} \mathbf{b}$ 表现最好，最高获 得 $65 \%$ 转化率和 $81 \%$ ee.

2016 年, Lefort 等[94]发现手性单齿亚磷酰胺配体 L28e 与非手性磷配体 $\mathrm{P}\left(p-\mathrm{CF}_{3} \mathrm{C}_{6} \mathrm{H}_{4}\right)_{3}$ 的异种组合在 $\mathrm{Ir}$ 催化氢化 2 位取代的吡定盐 $\mathbf{2 4}$ 中, 取得了非常好的结果 (Eq. 13). 这为手性环胺的合成提供了另一种有效途径. 


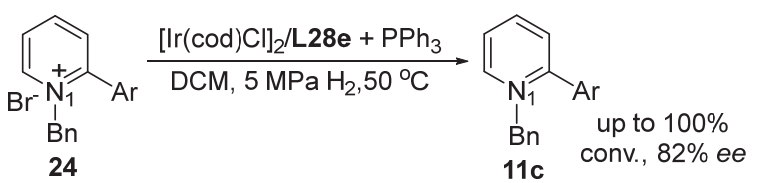

BINOL 亚磷酰胺配体的 $\mathrm{N}$ 原子上官能团化取代基 的影响也曾是一个热门研究领域. 2008 年报道的一篇综 述 ${ }^{[95]}$ 分别记载了含醚/硫醚、酯、酰胺、吡啶、叔胺、 亚磺酰亚胺的氨基对不对称氢化反应的影响, 并对单齿 亚磷酰胺的效果作了一个简单评估.

\subsection{Tropos 型配体}

Tropos亚磷酰胺配体也是一类诱导能力很强的配 体. 2005年, de Vries等[96]探索了Tropos联苯型磷配体在 组合化学和高通量篮选中的联合应用. 他们合成了 11 种 Tropos 亚磷酸酯配体和 8 种Tropos 亚磷酰胺配体, 将它 们用于 $\alpha$-脱氢氨基酸的不对称氢化中, 配体 L 36a 5 $\mathbf{L 3 6 b}$ (图10)的组合使用可使反应完全转化, 且产物的对 映选择性高达 $98 \%$ ee. 动力学研究表明, 亚磷酸酯配体 的手性诱导能力偏低, 但其对应的 $\mathrm{Rh}$ 催化剂的活性与 亚磷酸酯/亚磷酰胺组合使用更高; 而只含亚磷酰胺配 体的 $\mathrm{Rh}$ 配合物催化活性一般较低. 通过调配两者的用 量, 发现亚磷酸酯与亚磷酰胺的比例为 $0.25: 1.75$ 时, 催化效果最佳.

2007年, de Vries等[97]将含联苯骨架的单齿亚磷酰 胺配体用于 $I r$ 催化的 $\alpha$-脱氢氨基酸的不对称氢化中. 这 是首例含单齿配体的Ir配合物在不对称氢化中的应用, 也是首例仅包含一个单齿配体的金属配合物在不对称 氢化中的应用. 当增加配体联苯骨架上的 $3,3^{\prime}$ 取代基 $\mathrm{R}$ 的大小时, 转化率和对映选择性可以得到提高. 以底物 1 为例，当取代基为苯基时(L36c), ee值高达 $93 \%$, 而当 取代基为叔丁基时(L36d), 反应的对映选择性更好, 为 98\% eee. 此外, L36c 和 L 36d 在 $\beta$-位芳基或异丙基取代的 $\alpha$-脱氢氨基酸衍生物的不对称氢化中也表现优异, 获得 88\% 98\% ee, 但并不适用于 $\beta$ 位无取代的 $\alpha$-脱氢氨基 酸衍生物的不对称氢化, 对映选择性仅为 $39 \% \sim 50 \% e e$.

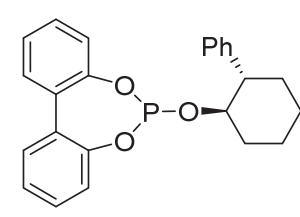

L36a

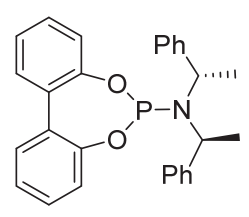

L36b

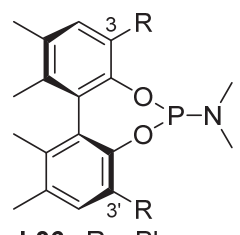

$\mathrm{L} 36 \mathrm{c} \mathrm{R}=\mathrm{Ph}$ L36d $R=t-B u$
图 10 优异的 Tropos 亚磷酰胺配体

Figure 10 Excellent Tropos phosphoramidite ligands

\subsection{DpenPhos 和 CydamPhos}

丁奎岭课题组报道了一系列 DpenPhos 配体和

CydamPhos配体及其在各类底物的不对称氢化中的应 用[98-101].

2005年，丁奎岭等 ${ }^{[98 a]}$ 设计合成了DpenPhos L37a (图11), 并将其用于 $\alpha$-脱氢氨基酸甲酯衍生物和 $\alpha$-芳基 烯酰胺的不对称氢化反应中，对映选择性均高于 $96 \%$

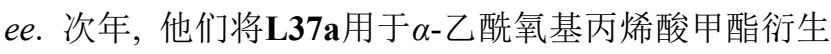
物 $25 \mathrm{a}$ 和 $\beta$-芳基衣康酸酯衍生物 $25 \mathrm{~b}$ 的不对称氢化反应 中, 但反应完全没有发生, 反而是 $\mathrm{N}$ 上含有活泼氢的一 类DpenPhos L37b表现极佳 ${ }^{[98 b]}$, 反应在室温下 10 h即可 完全转化, 产物的ee值都在 $94 \%$ 以上. 而且当催化剂用 量降低至 $0.025 \mathrm{~mol} \%, \beta$-芳基衣康酸酯衍生物的对映选 择性依旧高达 $97 \%$ ee. 他们提出，两个单齿亚磷酰胺配 体与金属配位以后，配体之间还存在着氢键的作用，这 种氢键作用使得催化剂具有极高的活性和不对称诱导 能力.

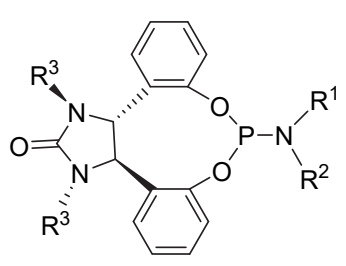

$(R, R)$-DpenPhos $(R, R)-\mathrm{L} 37 \mathrm{a} \mathrm{R}^{3}=\mathrm{Bn}, \mathrm{R}^{1}=\mathrm{R}^{2}=\mathrm{Me}$ $(R, R)-\mathrm{L} 37 \mathrm{~b} \mathrm{R}^{3}=\mathrm{R}^{1}=\mathrm{Bn}, \mathrm{R}^{2}=\mathrm{H}$

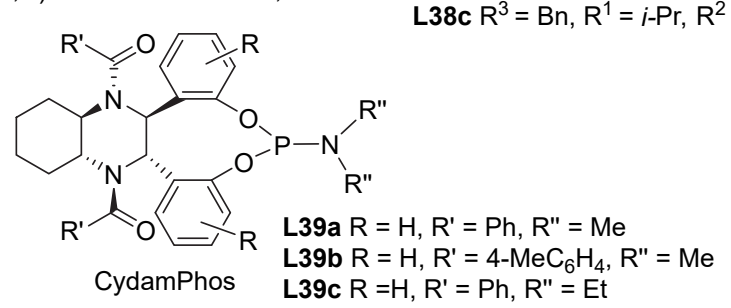

图 11 诱导效果好的的 DpenPhos 和 CyclamPhos 配体 Figure 11 Well-induced DpenPhos and CyclamPhos ligands

DpenPhos结构可调的特点便于将这类配体模块化 用于其他底物的不对称氢化中. 2011年, 丁奎岭课题 组 ${ }^{[98 c]}$ 设计了DpenPhos 型配体 L38 并用于 $R h$ 催化的烯酰 胺磷酸酯的不对称氢化中. 无论是 $\alpha$-烯酰胺磷酸酯 $26 \mathbf{a}$ 还是 $\beta$-烯酰胺磷酸酯 $\mathbf{2 6 b}$, 配体诱导的产物对映体过量 几乎都在 $95 \%$ 以上，当催化剂用量低至 $0.01 \mathrm{~mol} \%$ 时，可 以 $98 \% e e$ 获得强效氨肽酶抑制剂 $(R)-$ 磷亮氨酸的对映 体. 此外, 通过单晶衍射实验证明了催化前体包含有两 个配体同时与 $\mathrm{Rh} 中 心$ 配位，而非线性效应研究也印证 了参与催化循环的Rh活性物种可能包含有两个单齿配 体. 2012年, 该课题组又将DpenPhos型配体用于烯醇 酯、氧基取代的烯酸酯钾盐 $27^{[100]}$ 和 $\alpha, \beta$-不饱和膦酸酯 $\mathbf{2 8 a} \sim 28 b^{[101]}$ 的不对称氢化中, 反应几乎完全转化并获 得了出色的对映选择性(图12). 


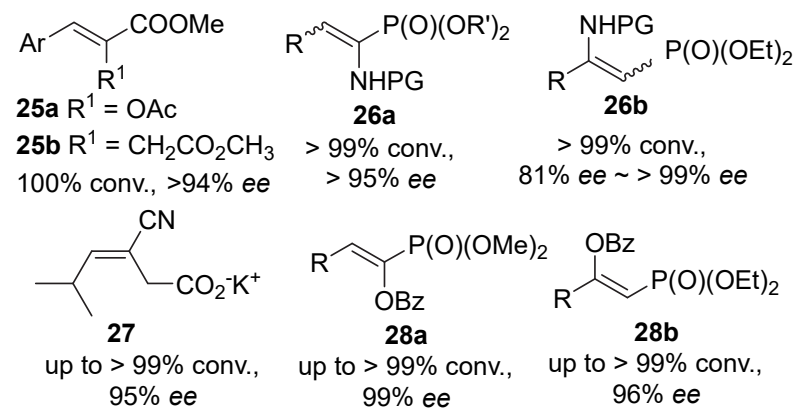

图 $12 \mathrm{Rh}$ 催化的使用 DpenPhos 诱导效果好的底物

Figure 12 Substrates with excellent ee induced by $\mathrm{Rh} /$ DpenPhos

但是 DpenPhos 制备较为复杂, 2006 年, 他们报道了 更易制备的 CydamPhos 型配体 L39 ${ }^{[99]}$. CydamPhos 具有 显著的特点: 原料廉价、制备简单、结构多样, 而且底 物适用性好, 在各类烯酰胺和 $\alpha$-脱氢氨基酸衍生物的不 对称氢化中均取得满意的结果 $(>90 \% e e)$; 此外, 改变 配体的取代基 $R 、 R^{\prime} 、 R$ "可以控制反应的立体选择性. 其 中 $\mathrm{R}^{\prime}$ 基团改可以改变产物的构型, 且当 $\mathrm{R}^{\prime}$ 基团为芳基 时，芳环上取代基越多，立体诱导效果越差; $\mathrm{R}$ "取代基

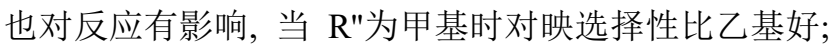
$\mathrm{R}$ 基团在苯环上的不同定位也会改变诱导能力 (ee 值)和 产物构型 $(R$ 或 $S$ 构型).

\section{4 螺环配体}

螺环骨架的刚性较大, 有利于不对称催化的立体选 择性的控制, 所以基于螺环骨架的亚磷酰胺配体也被大 量用于不对称氢化反应中, 周其林及其课题组在这一方 面做出了卓越的贡献.

2002 年, 周其林等[34]报道合成了螺环配体 SIPHOS (L40)(图 13), 将其用于 $\mathrm{Rh}$ 催化的 $\alpha$-脱氢氨基酸衍生物、 衣康酸二甲酯衍生物的不对称氢化中，反应均 $100 \%$ 转 化，且产物的对映选择性一般都在 $95 \%$ ee 以上. 这是首 例单齿螺环配体在不对称催化中的应用。后来，他们将 底物扩展到 $\alpha$-芳基烯酰胺 ${ }^{[33 a]}$ 和 $\beta$-脱氢氨基酸衍生 物 ${ }^{[33 b], ~} \mathbf{L 4 0}$ 表现出高于 $87 \% e e$ 的不对称诱导能力, 这是 首例单齿磷配体在烯酰胺的不对称氢化中的成功应 用[33a].

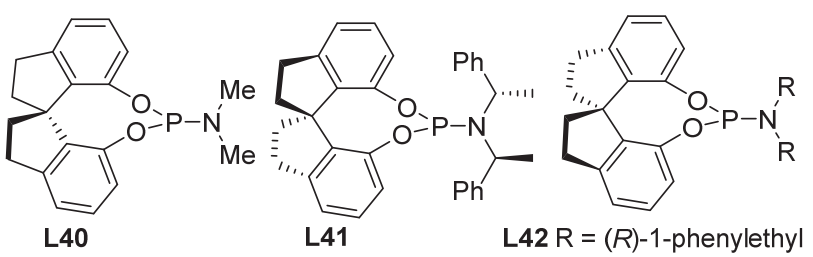

图 13 诱导效果好的手性螺环亚磷酰胺配体

Figure 13 Well-induced chiral spiro phosphoramidite ligands

2009 年, 他们报道了常压条件下螺环配体 $(R, S, S)-$
L41 在 Ir 催化的二氢吡咯类底物 29 的不对称氢化中的 应用 ${ }^{[102]}$, 以单质碘为添加剂, 四氢呋喃(THF)、二氧六 环(Dioxane)或乙二醇二甲醚(DME)作溶剂, 催化剂用量 为 $0.5 \mathrm{~mol} \%$ 时, 反应可 $100 \%$ 转化, 对映选择性均能达 到 $90 \%$ ee 以上. 考察不同类型的底物, 发现底物 $\alpha$-位的 基团 $\mathrm{R}^{1}$ 为芳基有利于获得高对映选择性. 进一步扩展 底物到六元环状烯胺和稠环烯胺 $30 \mathrm{a} \sim 30 \mathrm{~b}$, 前者氢化 所得产物的对映选择性只有 $21 \% \mathrm{ee}$, 后者则产生了较 为满意的效果，其中底物 $30 \mathbf{a}$ 的对映选择性为 $82 \% \mathrm{ee}$, 底物 30b 不对称氢化所得产物异喹啉生物碱 crispine A 的对映体过量高达 90\% (图 14).
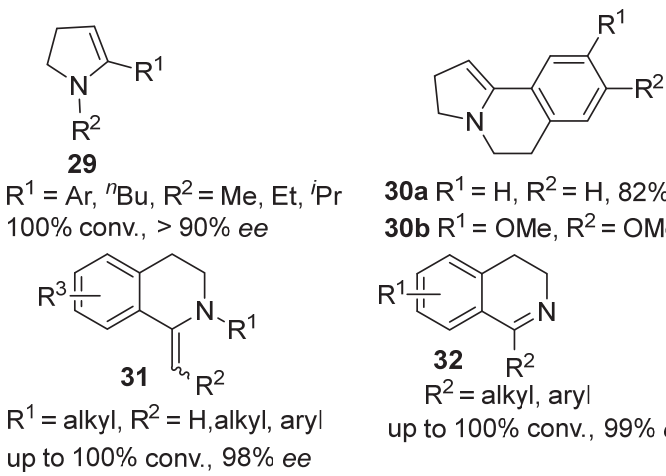

$30 a R^{1}=H, R^{2}=H, 82 \%$ ee $30 \mathrm{~b} \mathrm{R} \mathrm{R}^{1}=\mathrm{OMe}, \mathrm{R}^{2}=\mathrm{OMe}, 90 \%$ ee

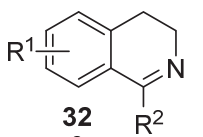

$\mathrm{R}^{2}=$ alkyl, aryl up to $100 \%$ conv., $99 \%$ ee

图 14 Ir 催化的使用单齿螺环配体诱导效果好的底物

Figure 14 Substrates with excellent $e e$ induced by chiral spiro iridium phosphoramidite complexes

同时，他们也研究了螺环配体催化环外双键烯胺 31 合成四氢异喹啉类化合物的反应 ${ }^{[103] ， 以 ~ L 42 ~}$ 为配体， 添加 $\mathrm{I}_{2}$ 或 $\mathrm{KI}$, 反应可完全转化, 对映选择性最高可达 $98 \%$ ee. 研究发现配体和金属配位的最佳比例为 $2: 1$, 推测在催化过程中, 催化循环中的活性 Ir 物种是含有两 个配体的，该物种在催化过程中逐渐形成，是反应获得 高对映选择性和高反应活性的关键. 结合相关实验数 据，他们提出 Ir 催化烯胺的不对称氢化可能经过 Ir 双氢 中间体的 $\operatorname{Ir}(\mathrm{I})-\mathrm{Ir}(\mathrm{III})$ 催化循环.

2012 年，周其林等 ${ }^{[104]}$ 实现了含螺环亚磷酰胺配体 的 Ir 配合物在环状亚胺 32 的催化氢化中的应用，用以 合成 1-烷基四氢异喹啉，该化合物是合成复杂生物碱的 重要中间体. 以 $\mathbf{L 4 1}$ 为配体, $\mathrm{Et}_{2} \mathrm{O} 、{ }^{t} \mathrm{BuOMe}$ 或甲苯作溶 剂, 添加碘单质或碘化物, 反应可完全转化, 对映选择 性高达 $99 \%$ ee . 另外, 若使用 KI 作添加剂, 即使氢气压 力降低至 $601.95 \mathrm{kPa}$, 也不影响转化率和对映选择性. 这类配体对多数环状亚胺都有高手性诱导能力, 无论底 物的 $\mathrm{R}^{2}$ 基团是烷基还是芳基，对映选择性保持在 $85 \%$ $e e$ 以上. 当 $\mathrm{R}^{2}$ 为烷基时，体积越大，相应的反应速率和 对映选择性越低; 且不论苯环上取代基 $\mathrm{R}^{1}$ 为吸电子还 是给电子取代基，反应的对映选择性较苯环未取代的底 物都会有轻微的降低，但是仍可保持在 $90 \%$ ee 以上. 


\section{5 其他类型单齿亚磷酰胺配体}

2015 年, Franciò 等 ${ }^{[105]}$ 报道了含手性 P 原子的亚磷 酰胺配体 $\mathrm{L43}$ 在 $\mathrm{Rh}$ 催化衣康酸二甲酯 $\mathbf{2}$ 的不对称氢化 中的应用，当 $\mathrm{X}$ 基团为 $\mathrm{OCH}_{3} 、 \mathrm{P}$ 原子为 $S$ 构型、催化 剂转化数 $S / C$ 为 1000 时, 效果最好, 反应几乎完全转化 并获得 $85 \%$ ee 的产物.<smiles>[X]P1Oc2ccc3ccccc3c2C(c2ccccc2)N1[C@@H](C)c1ccccc1</smiles>

$\mathrm{L} 43 \mathrm{X}=\mathrm{OR}, \mathrm{NR}_{2}$

\section{5 单齿磷配体的延伸与应用}

\section{1 超分子配体}

单齿配体通过非共价键或离子相互作用自组装成 二齿超分子配体，它们既有二齿配体刚性大等重要特 征, 又保留了单齿配体原子经济性等优点. 这类配体与 过渡金属形成的配合物类似于金属酶, 可将底物结合在 金属中心附近, 实现底物的高效催化转化, 是近年来配 体开发领域里备受关注的新方向.

2007 年, Nishibayashi 小组 ${ }^{[106]}$ 成功将类轮烷分子作 为手性配体用于均相过渡金属催化中. 他们报道了含有 冠醚骨架的超分子手性亚磷酸酯配体 L46. 将 L46 用于 $\alpha$-脱氢氨基酸酯的不对称氢化反应中, 反应可以获得 $99 \%$ 以上的转化率和 $90 \%$ 以上 $e e$ 值. 当只有 $\mathbf{L 4 4}$ 诱导 该反应时, 反应活性和选择性都很差, 仅当 $\mathrm{L44}$ 与 $\mathbf{L 4 5}$ 两种配体同时使用才能取得最好的结果, 可见这种类轮 烷超分子的形成对提高催化活性和对映选择性有明显 帮助. 此外, 如果类轮烷的亚磷酸酯部分和膦部分距离 过远, 则无法与 $\mathrm{Rh}$ 中心同时配位, 即无法发挥协同催 化作用. 次年, 范青华课题组 ${ }^{[107]}$ 则将类似的类轮烷配 体用于一系列 $\alpha$-脱氢氨基酸酯的不对称氢化中, 对映选 择性最高达 $84 \%$ ee.

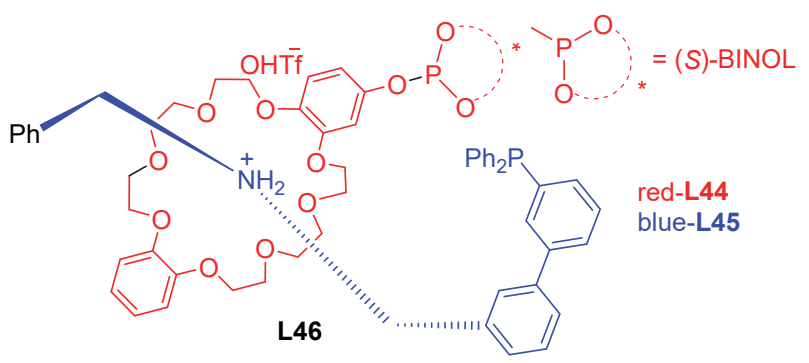

2009 年, Gennari 等 ${ }^{[108]}$ 将含羧酸和苠胺的 BINOL 单 齿亚磷酸酯配体通过酸碱相互作用自组装为超分子配 体，成功催化了 $\alpha$-乙酰氨基丙烯酸甲酯 3 的不对称氢化, 底物实现了完全转化, 产物 $e e$ 值高达 $90 \% .2010$ 年, 他
们将邻苯二甲酰胺和 BINOL 单齿亚磷酸酯通过氨基醇 间隔基(linker)连接起来, 组装为 Phthalaphos ${ }^{[109]}$ (图 15), 两个单齿配体与 $\mathrm{Rh}$ 形成配位化合物，配体之间通过氢 键相连, 整个配合物 34 以双齿的形式表现出来(图 15), 无论是标准底物 3 和 $\mathbf{4}$, 还是挑战性底物环状烯酰胺 6 、 $\beta^{2}$-脱氢氨基酸酯 33, 均获得了优异的立体选择性(Eq. 14). 在此研究基础上, 他们进一步探究了氢键的具体 作用 ${ }^{[110]}$, 推测在催化循环中, 超分子配合物 34 的氢键 会断开, 取而代之的是底物酰胺 NH 原子与配体的酰胺 $\mathrm{O}$ 原子形成氢键, 使底物以固定的角度与金属中心进行 配位，从而形成单一中间体 35 (Scheme 4), 这样也就解 释了反应速率和对映选择性高的原因. 将 Phthalaphos 配体的邻苯二甲酰胺用苯甲酰胺替代, 即得到 Benzaphos 配体(图 15), 它在脱氢氨基酸酯和烯酰胺的 不对称氢化中也表现出良好的不对称诱导活性 ${ }^{[111]}$.

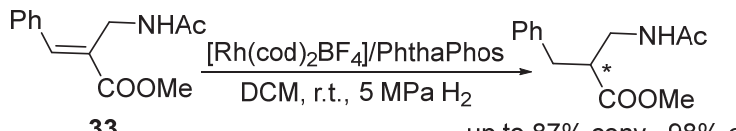

up to $87 \%$ conv., $98 \%$ ee

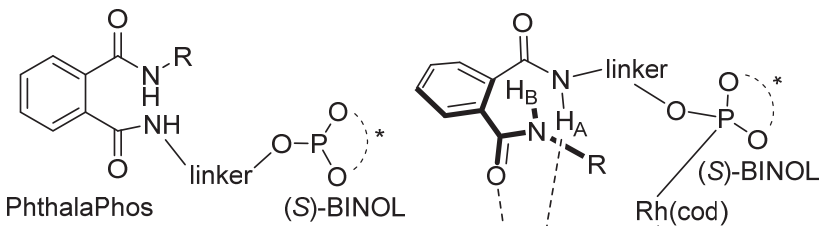
PhthalaPhos (S)-BINOL $\quad: \quad$ Rh(cod) $\mathrm{R}=p-{ }^{n} \mathrm{Bu}-\mathrm{C}_{6} \mathrm{H}_{4}, 3,5-\left(\mathrm{CF}_{3}\right) \mathrm{C}_{6} \mathrm{H}_{3},{ }^{n} \mathrm{Bu}$

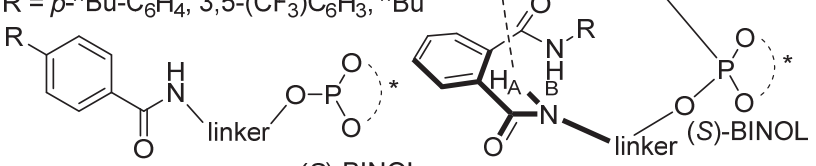

BenzaPhos $\mathrm{R}=\mathrm{H}, \mathrm{CF}_{3}, \mathrm{OMe}$
(S)-BINOL
图 15 PhthaPhos、 benzaPhos 以及由 PhthaPhos 组装的超分子 配合物

Figure 15 PhthaPhos ligand, benzaPhos ligand and the supramolecular complex based on PhthalaPhos

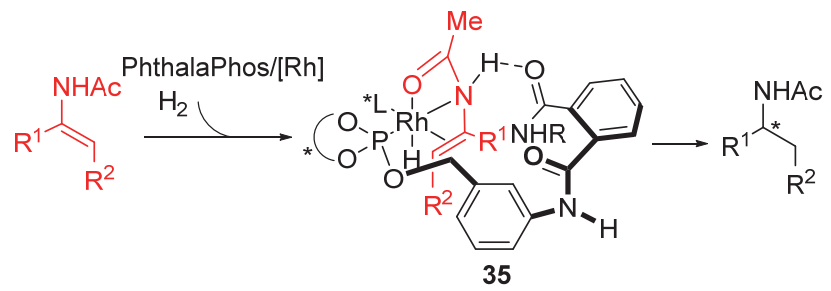

图式 4 通过和 PhthaPhos 配体形成氢键实现底物的定向定位 Scheme 4 Substrate orientation by forming hydrogen bonding with PhthaPhos ligand

2009 年，范青华课题组 ${ }^{[112]}$ 将单齿亚磷酸酯配体通 过氢键作用针定在树突状载体上得到了树突状手性超 分子配体, 并将其用于烯酰胺和脱氢氨基酸衍生物的不 对称氢化中，反应完全转化，产物对映选择性高达 $91 \%$ 
$e e$. 该催化剂可以通过溶剂沉淀的方法回收利用, 且循 环利用 5 次后催化能力依旧没有明显的降低.

2010 年, Breit 等[113]等将手性单齿磷配体的多样性 合成、氢键自组装以及迭代组合篮选相结合，突破了 HTS 的瓶颈, 如配体库的有限以及配体篎选的巨大工作 量. Breit 设计的 $\mathrm{AD}($ 氢键受体一给体 $)$ 型与 $\mathrm{DA}($ 氢键给体受体)型单齿磷配体之间通过形成氢键实现分子自组装. 他们合成了 $6+6$ 种 $\mathrm{AD}$ 型配体和 $5+5$ 种 $\mathrm{DA}$ 型配体, 这 些配体有 $120(12 \times 10)$ 种组合方式形成超分子配体, 将 它们分为 4 个亚组并与 $\mathrm{Rh}$ 前体混合, 将每个亚组的超 分子配合物的混合物用于 $\alpha$-乙酰氨基丙烯酸甲酯 $\mathbf{3}$ 的不 对称氢化中, 选出平均诱导效果最佳的亚组, 然后将该 亚组继续迭代分组, 直到选出最佳配体组合, 最佳组合 分别为 $L 47 a / L 48 a 、 L 47 a / L 48 b 、 L 47 b / L 48 a$, 反应均可 实现完全转化且 $e e$ 值达 99\% (Eq. 15). 这和 120 种组合 平行篎选方法的结果是一致的, 而且该方法仅进行了 17 次实验, 可见迭代法在笁选最佳配体组合时是非常 实用的.
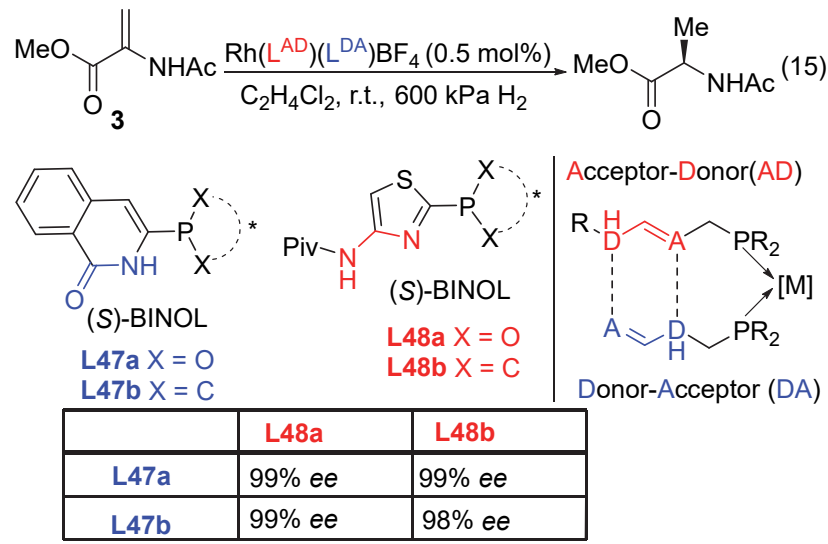

\section{2 催化剂的回收利用}

前面提到，单齿磷配体易合成，原料低廉，由此也 衍生出了很多运用, 如配体的高通量篮选, 超分子配 体、组合配体的使用等. 但其自身也面临着一些问题, 例如配位金属贵重, 均相催化条件下催化剂难以实现回 收再循环使用. 为此, 化学家们做出了各种尝试.

2003 年, Doherty 等[114]将亚磷酰胺负载于可溶性非 交联聚苯乙烯上, 得到了大分子配体 L49, 但负载后的 催化剂性能较未负载的而言有明显的降低, 而且该配体 的固载过程步骤繁琐，很大程度上提高了制备成本.

为了避免对配体进行复杂结构修饰而引起的不对 称诱导能力降低, 2004 年, Sheldon 等[115]提出催化剂 $\mathrm{Rh} /$ L28a 通过离子交换作用固载在铝硅酸盐 AlTUD-1 上形成 $\mathbf{R h} / \mathbf{L} 28 \mathbf{a}-A I T U D-1$, 它被成功用于水作溶剂的 $\alpha$-乙酰氨基丙烯酸甲酯 3 的非均相不对称氢化中，产物 的 $e e$ 值高达 $95 \%$, 且回收 4 次后，活性和选择性几乎不 变. 但使用异丙醇作溶剂时, 由于催化剂大量浸出, 导 致催化剂活性明显降低. 这样将催化剂固载在惰性载体 上的方法有可能使催化剂无规则针定在载体上, 造成催 化剂活性和选择性在一定程度上有所降低.

2004 年, 丁奎岭等[116]提出了非均相催化剂的自负 载, 他们将桥联的手性单齿亚磷酰胺配体 MonoPhos 与 $\mathrm{Rh}$ 结合，自组装成具有永久多孔性兼具吸附有机分子 能力的金属-有机配位聚合物 36, 在 $\alpha$-脱氢氨基酸和烯 酰胺的不对称氢化中，该自负载催化剂实现了底物的完 全转化, 立体诱导能力甚至婫美均相催化剂, 最高达 $97.3 \% e e$, 且催化剂回收 6 次后，其活性保持不变，选择 性仍在 $90 \%$ ee 以上.

2006 年，郑卓等 ${ }^{[117]}$ 合成了可溶性 MeOPEG-单齿亚 磷酸酯配体 L50a L50c (图 16), 它们在 Rh 催化烯酰胺 和 $\beta$-脱氢氨基酸酯的不对称氢化中表现出优异的手性 诱导能力 $(93 \% \sim 98 \% e e)$. 而且, 此类催化剂循环使用 四次后，催化活性和选择性仍能基本保持. 但是这类配 体在空气或水中不稳定，还需要进一步改良.
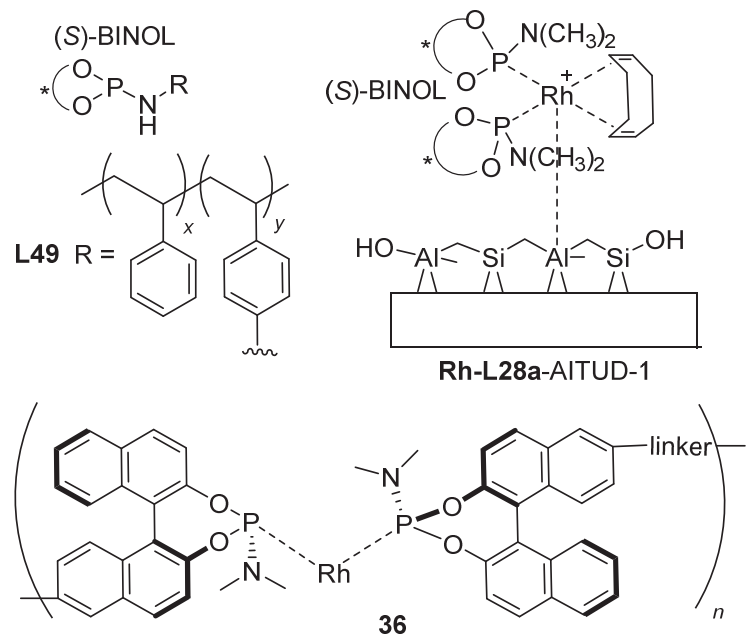

(S)-BINOL
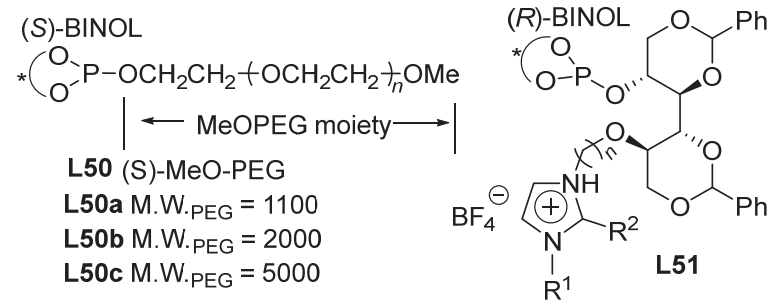

图 16 催化剂回收利用的方法

Figure 16 Methods for recycling catalyst

2011 年, Huang 等[118]和 Escárcega-Bobadilla 等[119] 相继报道了离子型亚磷酸酯配体的回收利用, 前者将 L51 与 Rh 形成的配合物溶于离子液体一甲苯两相溶剂系 统中, 即使回收次数超过 10 次，催化活性也没有明显的 
降低. 后者则以失败告终, 无论是把催化剂溶于离子液 体 $[\mathrm{BMI}]\left[\mathrm{PF}_{6}\right]$ 中, 还是溶解后再负载于碳纳米管上, 回 收后的催化剂均失去诱导活性.

2014 年, 赵耀鹏课题组 ${ }^{[120]}$ 提出了通过吸附法实现 Rh-MonoPhos 的回收利用, 他们借助多孔硅胶对 $\mathrm{Rh}$ 催 化剂的强亲和力以及 Rh-MonoPhos 在环已烷中的低溶 解度, 以环己烷为溶剂, 将 Rh-MonoPhos 催化剂吸附于 多孔笼状二氧化硅上, 随后应用于催化衣康酸二甲酯 $\mathbf{2}$ 等底物的不对称氢化, 反应的转化率和对映选择性均比 使用 Rh-MonoPhos 更高; 且催化剂回收 4 次后活性和选 择性仅轻微降低.

\section{6 结论与展望}

毫无疑问, 不对称催化一直是有机化学领域的热点 课题, 其强大的活力源自于广泛的工业应用前景和巨大 的市场. 手性配体和手性催化剂的设计是不对称催化研 究中永恒的课题, 一方面的原因是没有一种配体或催化 剂是通用的, 另一方面, 许多优秀的配体和催化剂都已 被专利保护, 其工业应用受到了很大限制, 因此发展新 型手性配体和催化剂仍是未来的重要任务之一.

由于单齿亚磷酸酯配体稳定性好、合成简单、原料 价格低廉, 该类配体与金属形成的催化体系在催化不饱 和键的不对称氢化中表现出优异的催化活性和立体选 择性, 其催化效率和对映选择性可以与目前最好的双膦 手性配体如 BINAP, DIPAMP 等形成的催化剂相妶美, 因此单齿亚膦酸酯、亚磷酸酯、亚磷酰胺类配体在工业 化中有很大的应用潜力. 继续这一领域的探索, 开发稳 定性好、适用范围广的新型催化体系, 无论是在学术研 究还是在工业应用方面都有重要的意义和较大的指导 作用. 我们有理由相信, 在不远的将来会有更多结构新 颖并且性能优异的手性磷配体出现.

\section{References}

[1] Blaser, H. U.; Schmidt, E. In Asymmetric Catalysis on Industrial Scale, Wiley, New York, 2004.

[2] (a) Chu, W.; Guo, F.; Yu, L.; Hong, J.; Liu, Q.; Mo, F.; Zhang, Y.; Wang, J. Chin. J. Chem. 2018, 36, 217.

(b) Yang, Y.; L. H.; Liu, X.; Liu, T.; Zhu, Y.; Zhang, A.; Mao, G.; Liu, L. Chin. J. Org. Chem. 2019, 39, 1655 (in Chinese).

(杨云, 刘慧慧, 刘小兵, 刘田田, 朱玉芹, 张安安, 毛国梁, 刘 澜涛, 有机化学, 2019, 39, 1655.)

(c) Xu, R.; Yang, P.; Zheng, C.; You, S. Chin. J. Chem. 2020, 38, 683.

(d) Xing, A.; Tian, M.; Wang, L. Chin. J. Org. Chem. 2016, 36, 2912 (in Chinese).

(邢爱萍, 田密, 王来来, 有机化学, 2016, 36, 2912.)

(e) Zhang, F.; Liu, X.; Liu, W.; Deng, G. Chin. J. Org. Chem. 2017, 37, 2555 (in Chinese).

(张凤, 刘祥华, 刘玮, 邓国军, 有机化学, 2017, 37, 2555.)

[3] Claver, C.; Fernandez, E.; Gillon, A.; Heslop, K.; Hyett, D. J.; Martorell, A.; Orpen, A. G.; Pringle, P. G. Chem. Commun. 2000, 961.
[4] Reetz, M. T.; Mehler, G. Angew. Chem., Int. Ed. 2000, 39, 3889.

[5] van den Berg, M.; Minnaard, A. J.; Schudde, E. P.; van Esch, J.; de Vries, A. H. M.; de Vries, J. G.; Feringa, B. L. J. Am. Chem. Soc. 2000, 122, 11539 .

[6] Fu, W.; Tang, W. ACS Catal. 2016, 6, 4814.

[7] (a) Xia, J.; Nie, Y.; Yang, G.; Liu, Y.; Gridnev, I. D.; Zhang, W. Chin. J. Chem. 2018, 36, 612.

(b) Ma, X.; Qiao, L.; Liu, G.; Huang, Z. Chin. J. Chem. 2018, 36, 1151.

(c) Jia, X.; Ren, X.; Wang, Z.; Xia, C.; Ding, K. Chin. J. Org. Chem. 2019, 39, 207 (in Chinese).

(贾肖飞, 任新意, 王正, 夏春谷, 丁奎岭, 有机化学, 2019, 39, 207.)

(d) Li, S.; Li, Z.; You, C.; Lü, H.; Zhang, X. Chin. J. Org. Chem. 2019, 39, 1568 (in Chinese).

(李帅龙, 李庄星, 由才, 吕辉, 张绪穆, 有机化学, 2019, 39, 1568.)

(e) Chen, D.; Xu, M. Chin. J. Org. Chem. 2017, 37, 1589 (in Chinese).

(陈雕, 徐明华, 有机化学, 2017, 37, 1589.)

(f) Yu, Y.; Xu, M. Acta Chim. Sinica, 2017, 75, 655 (in Chinese). (于月娜, 徐明华, 化学学报, 2017, 75, 655.)

[8] Rössler, S. L.; Petrone, D. A.; Carreira, E. M. Acc. Chem. Res. 2019, 52, 2657.

[9] (a) Knowles, W. S.; Sabacky, M. J. Chem. Commun. (London) 1968, 22, 1445.

(b) Knowles, W. S.; Sabacky, M. J.; Vineyard, B. D. J. Chem. Soc., Chem. Commun. 1972, 1, 10.

(c) Vineyard, B. D.; Knowles, W. S.; Sabacky, M. J.; Bachman, G. L.; Weinkauff, D. J. J. Am. Chem. Soc. 1977, 99, 5946.

[10] Knowles, W. S. Acc. Chem. Res. 1983, 16, 106.

[11] Horner, L.; Siegel, H.; Büthe, H. Angew. Chem., Int. Ed. Engl. 1968, 7, 942.

[12] Dang, T. P.; Kagan, H. B. J. Chem. Soc.: Chem. Commun. 1971, 10, 481.

[13] Dang, T. P.; Kagan, H. B. J. Am. Chem. Soc. 1972, 94, 6429.

[14] Reetz, M. T.; Sell, T. Tetrahedron Lett. 2000, 41, 6333.

[15] Minnaard, A. J.; Feringa, B. L.; Lefort, L.; de Vries, J. G. Acc. Chem. Res. 2007, 40, 1267.

[16] Reetz, M. T.; Meiswinkel, A.; Mehler, G.; Angermund, K.; Graf, M.; Thiel, W.; Mynott, R.; Blackmond, D. G. J. Am. Chem. Soc. 2005, 127, 10305.

[17] Reetz, M. T.; Sell, T.; Meiswinkel, A.; Mehler, G. Angew. Chem., Int. Ed. 2003, 42, 790 .

[18] Reetz, M. T., Mehler, G. Tetrahedron Lett. 2003, 44, 4593.

[19] Pena, D.; Minnaard, A. J.; Boogers, J. A. F.; de Vries, A. H. M.; de Vries, J. G.; Feringa, B. L. Org. Biomol. Chem. 2003, 1, 1087.

[20] Hoen, R.; Boogers, J. A. F.; Bernsmann, H.; Minnaard, A. J.; Meetsma, A.; Tiemersma-Wegman, T. D.; de Vries, A. H. M.; de Vries, J. G.; Feringa, B. L. Angew. Chem., Int. Ed. 2005, 44, 4209.

[21] Reetz, M. T.; Li, X. Chem. Commun. 2006, 2159.

[22] Breit, B.; Seiche, W. J. Am. Chem. Soc. 2003, 125, 6608.

[23] Breit, B; Seiche, W. Angew. Chem., Int. Ed. 2005, 44, 1640.

[24] Breit, B. Angew. Chem., Int. Ed. 2005, 44, 6816.

[25] Takacs, J. M.; Reddy, D. S.; Moteki, S. A.; Wu, D.; Palencia, H. J. Am. Chem. Soc. 2004, 126, 4494.

[26] (a) Takacs, J. M.; Chaiseeda, K.; Moteki, S. A.; Reddy, D. S.; Chandra, D.; Wu, K. Pure Appl. Chem. 2006, 78, 501.

(b) Moteki, S. A.; Toyama, K.; Liu, Z.; Ma, J.; Holmes, A. E.; Takacs, J. M. Chem. Commun. 2012, 263.

[27] (a) Slagt, V. F.; van Leeuwen, P. W. N. M.; Reek, J. N. H. Chem. Commun. 2003, 19, 2474.

(b) Kuil, M.; Goudriaan, P. E.; van Leeuwen, P. W. N. M.; Reek, J. N. H. Chem Commun, 2006, 45, 4679.

(c) Jiang, X.-B.; van Leeuwen, P. W. N. M; Reek, J. N. H. Chem. Commun. 2007, 22, 2287.

[28] Frank, D. J; Franzke, A.; Pfaltz, A. Chem.-Eur. J. 2013, 19, 2405.

[29] Lefort, L.; Boogers, J. A. F.; de Vries, A. H. M.; de Vries, J. G. Org. 
Lett. 2004, 6, 1733.

[30] Lefort, L.; Boogers, J. A. F.; Kuilman, T.; Vijn, R. J.; Janssen, J.; Straatman, H. Org. Process Res. Dev. 2010, 14, 568.

[31] Alexakis, A.; Rosset, S.; Allamand, J.; March, S.; Guillen, F.; Benhaim, C. Synlett 2001, 1375.

[32] Wunnemann, S.; Frohlich, R.; Hoppe, D. Org. Lett. 2006, 8, 2455.

[33] (a) Hu, A.-G.; Fu, Y.; Xie, J.-H.; Zhou, H.; Wang, L.-X; Zhou, Q.-L. Angew. Chem., Int. Ed. 2002, 41, 2348.

(b) Fu, Y.; Guo, X.-X.; Hu, A.-G.; Zhou, Q.-L. J. Org. Chem. 2004, 69,4648 .

[34] Fu, Y.; Xie, J.-H.; Hu, A.-G.; Zhou, H.; Wang, L.-X.; Zhou, Q.-L. Chem. Commun. 2002, 5, 480

[35] Zhu, S.-F.; Fu, Y.; Xie, J.-H.; Liu, B.; Xing, L.; Zhou, Q.-L. Tetrahedron: Asymmetry 2003, 14, 3219.

[36] Fleming, J. T.; Wills, C.; Waddell, P. G.; Harrington, R. W.; Higham, L. J. Dalton Trans. 2016, 45, 15660.

[37] Fu, Y.; Hou, G.-H.; Xie, J.-H.; Xing, L.; Wang, L.-X.; Zhou, Q.-L. J. Org. Chem. 2004, 69, 8157.

[38] Hou, G.-H.; Xie, J.-H.; Wang, L.-X.; Zhou, Q.-L. J. Am. Chem. Soc. 2006, 128, 11774.

[39] Xu, Y. J.; Docherty, G. F.; Woodwardc, G.; Wills M. Tetrahedron: Asymmetry 2006, 17, 2925.

[40] van Leeuwen, P. W. N. M; Kamer, P. C. J.; Claver, C.; Pamies, O.; Dieguez, M. Chem. Rev. 2011, 111, 2077.

[41] Diéguez, M.; Pàmies, O.; Claver, C. Tetrahedron: Asymmetry 2004, $15,2113$.

[42] Alexakis, A.; Bäckvall, J. E.; Krause, N.; Pàmies, O.; Diéguez, M. Chem. Rev. 2008, 108, 2796.

[43] Reetz, M. T.; Mehler, G.; Meiswinkel, A.; Sell, T. Tetrahedron Lett. 2002, 43, 7941

[44] Chen, W.; Xiao, J. Tetrahedron Lett. 2001, 42, 2897.

[45] Jerphagnon, T.; Renaud, J. L.; Demonchaux, P.; Ferreira, A.; Bruneau, C. Adv. Synth. Catal. 2004, 346, 33.

[46] Lyubimov, S. E.; Davankov, V. A.; Loim, N. M.; Popova, L. N.; Petrovskii, P. V.; Valetskii, P. M.; Gavrilov, K. N. J. Organomet. Chem. 2006, 691, 5980.

[47] Lynikaite, B.; Cvengros, J.; Piarulli, U.; Gennari, C. Tetrahedron Lett. 2008, 49, 755.

[48] Lyubimov, S. E.; Tyutyunov, A. A.; Kalinin, V. N.; Said-Galiev, E. E.; Khokhlov, A. R.; Petrovskii, P. V. Tetrahedron Lett. 2007, 48, 8217.

[49] Lyubimov, S. E.; Kalinin, V. N.; Tyutyunov, A. A.; Olshevskaya, V. A.; Dutikova, Y. V.; Safranov, A. S.; Davankov, V. A. Chirality 2009, 21, 5 .

[50] Lyubimov, S. E.; Kuchurov, I. V.; Tyutyunov, A. A.; Petrovskii, P. V.; Kalinin, V. N.; Zlotin, S. G.; Davankov, V. A.; Hey-Hawkins, E. Catal. Commun. 2010, 11, 419.

[51] Lyubimov, S. E.; Ozolin, D. V.; Davankov, V. A. Tetrahedron Lett. 2014, 55, 3613.

[52] Au-Yeung, T. T.-L.; Chan, S.-S.; Chan, A. S. C. Adv. Synth. Catal. 2003, 345, 537.

[53] Gergely, I.; Hegedüs, C.; Gulyás, H.; Szöllősy, Á.; Monsees, A.; Riermeier, T. Tetrahedron: Asymmetry 2003, 14, 1087.

[54] Reetz, M. T.; Ma, J.-A.; Goddard, R. Angew. Chem., Int. Ed. 2005, 44, 412.

[55] Dai, Y.; Yang, G.; Zhang, Y.; Zhang, W. Chin. J. Org. Chem. 2008, 28, 1169 (in Chinese). (戴轶俊, 杨国强, 张勇健, 张万斌, 有机化学, 2008, 28, 1169.)

[56] Chen, W.; Xiao, J. Tetrahedron Lett. 2001, 42, 8737.

[57] Hannen, P.; Militzer, H.-C.; Vogl, E. M. Chem. Commun. 2003, 2210 .

[58] Iuliano, A.; Losi, D.; Facchetti S. J. Org. Chem. 2007, 72, 8472.

[59] Reetz, M. T.; Li, X. Angew. Chem., Int. Ed. 2005, 44, :2959.

[60] Reetz, M. T.; Goossen, L. J.; Meiswinkel, A.; Paetzold, J.; Jensen, J. F. Org. Lett. 2003, 5, 3099.

[61] Huang, H.; Zheng, Z.; Luo, H.; Bai, C.; Hu, X.; Chen, H. Org. Lett. 2003, 5,4137 .

[62] (a) Huang, H.; Liu, X.; Chen, S.; Chen, H.; Zheng, Z. Tetrahedron:
Asymmetry 2004, 15, 2011

(b) Huang, H.; Zheng, Z.; Luo, H.; Bai, C.; Hu, X.; Chen, H. J. Org. Chem. 2004, 69, 2355.

[63] (a) Huang, H.; Liu, X.; Chen, H.; Zheng, Z. Tetrahedron: Asymmetry 2005, 16, 693 .

(b) Huang, H.; Liu, X.; Deng, J.; Qiu, M.; Zheng, Z. Org. Lett. 2006, 8,3359 .

[64] Alegre, S.; Alberico, E.; Pàmies, O.; Diéguez, M. Tetrahedron: Asymmetry 2014, 25, 258.

[65] Barakat, A.; Al-Majid, A. M. Arab. J. Chem. 2017, 10, S894.

[66] Reetz, M. T.; Guo, H.; Ma, J.-A.; Goddard, R.; Mynott, R. J. J. Am. Chem. Soc. 2009, 131, 4136.

[67] Carrilho, R. M. B.; Costa, G. N.; Neves, Â. C. B.; Pereira, M. M.; Grabulosa, A.; Bayón, J. C. Eur. J. Inorg. Chem. 2014, $2014,1034$.

[68] Zhu, S.-F.; Liu, T.; Yang, S.; Song, S.; Zhou, Q.-L. Tetrahedron 2012, 68, 7685.

[69] Hu, X.-P.; Wang, D.-S.; Yu, C.-B.; Zhou, Y.-G.; Zheng, Z.; Ma, S., Adventure in Asymmetric Hydrogenation: Synthesis of Chiral Phosphorus Ligands and Asymmetric Hydrogenation of Heteroaromatics, Springer-Verlag Berlin Heidelberg, 2011, Vol. 36, p. 2557.

[70] Yuan, Q.; Zhang, W. Chin. J. Org. Chem. 2016, 36, 274 (in Chinese).

(袁乾家，张万斌，有机化学, 2016, 36, 274.)

[71] Hulst, R.; de Vries, N. K.; Feringa, B. L. Tetrahedron: Asymmetry 1994, 5,699 .

[72] de Veris, A. H. M.; Meetsma, A.; Feringa, B. L. Angew. Chem., Int. Ed. Engl. 1996, 35, 2374.

[73] van den Berg, M.; Minnaard, A. J.; Haak, R. M. Adv. Synth. Catal. 2003, 345, 308.

[74] Jia, X.; Guo, R.; Li, X. Tetrahedron. Lett. 2002, 43, 5541.

[75] Peña, D.; Minnaard, A. J.; de Vries, J. G.; Feringa, B. L. J. Am. Chem. Soc. 2002, 124, 14552.

[76] Jia, X.; Li, X.; Xu, L. J. Org. Chem. 2003, 68, 4539

[77] Zeng, Q.; Liu, H.; Cui, X. Tetrahedron: Asymmetry 2002, 13, 115.

[78] Zeng, Q.; Liu, H.; Mi, A. Tetrahedron 2002, 58, 8799.

[79] Erre, G.; Junge, K.; Enthaler, S.; Addis, D.; Michalik, D.; Spannenberg, A.; Beller, M. Chem.-Asian J. 2008, 3, 887.

[80] Enthaler, S.; Erre, G.; Junge, K.; Schröder K, Addis D, Michalik D. Eur. J. Org. Chem. 2008, 3352.

[81] Erre, G.; Enthaler, S.; Junge, K.; Addis, D.; Beller, M. Adv. Synth Catal. 2009, 351, 1437.

[82] Bernsmann, H.; van den Berg, M.; Hoen, R.; Minnaard, A. J.; Mehler, G.; Reetz, M. T. J. Org. Chem. 2005, 70, 943.

[83] Panella, L.; Aleixandre, A. M.; Kruidhof, G. J.; Robertus, J.; Feringa, B. L.; de Vries, J. G. J. Org. Chem. 2006, 71, 2026.

[84] Panella, L.; Feringa, B. L.; de Vries, J. G. Org. Lett. 2005, 7, 4177.

[85] Hekking, K. F. W.; Lefort, L.; de Vries, A. H. M.; van Delft, F. L.; Schoemaker, H. E.; de Vries, J. G. Adv. Synth. Catal. 2008, 350, 85.

[86] Feringa, B. L.; de Vries, J. G. Adv. Synth. Catal. 2008, 350, 1081.

[87] Feringa, B. L.; de Vries, J. G. Adv. Synth. Catal. 2009, 351, 2549.

[88] Feringa, B. L.; de Vries, J. G. Tetrahedron: Asymmetry 2010, 21, 7.

[89] Mršić, N.; Minnaard, A. J.; Feringa, B. L.; Vries, J. G. D. J. Am. Chem. Soc. 2009, 131, 8358.

[90] Eberhardt, L.; Armspach, D.; Matt, D.; Toupet, L.; Oswald, B. Eur. J. Org. Chem. 2007, 2007, 5395 .

[91] Eberhardt, L.; Armspach, D.; Matt, D.; Toupet, L.; Oswald, B. Eur. J. Inorg. Chem. 2007, 26, 4153.

[92] Lyubimov, S. E.; Rastorguev, E. A.; Verbitskaya, T. A.; Petrovskii, P. V.; Hey-Hawkins, E.; Kalinin, V. N.; Davankov, V. A. Polyhedron 2011, 30, 1258.

[93] Schmitz, C.; Leitner, W.; Franciò, G. Eur. J. Org. Chem. 2015 , 2889.

[94] Renom-Carrasco, M.; Gajewski, P.; Pignataro, L.; de Vries, J. G.; Piarulli, U.; Gennari, C. Adv. Synth. Catal. 2016, 358, 2589.

[95] Eberhardt, L.; Armspach, D.; Harrowfield, J.; Matt, D. Chem. Soc. Rev. 2008, 37, 839.

[96] Monti, C.; Gennari, C.; Piarulli, U.; de Vries, J. G.; de Vries, A. H. M.; Lefort, L. Chem.-Eur. J. 2005, 11, 6701. 
[97] [97] Giacomina, F.; Meetsma, A.; Panella, L.; Lefort, L.; de Vries, A. H. M.; de Vries, J. G. Angew. Chem., Int. Ed. 2007, 46, 1497.

[98] (a) Liu, Y.; Ding, K. J. Am. Chem. Soc. 2005, 127, 10488. (b) Liu, Y.; Sandoval, C. A.; Yamaguchi, Y.; Zhang, X.; Wang, Z.; Kato, K.; Ding, K. J. Am. Chem. Soc. 2006, 128, 14212. (c) Zhang, J. Z.; Li, Y.; Wang, Z.; Ding, K. Angew. Chem., Int. Ed. 2011, 50, 11743 .

(d) Zhang, J. Z.; Dong, K.; Wang, Z.; Ding, K. Org. Biomol. Chem. 2011, 11,1598 .

(e) Liu, Y.; Wang, Z.; Ding, K. Acta Chim. Sinica 2012, 70, 1464 (in Chinese).

(刘龍, 王正, 丁奎岭, 化学学报, 2012, 70, 1464.)

[99] Zhao, B.; Wang, Z.; Ding, K. Adv. Synth. Catal. 2006, 348, 1049.

[100] Liu, Y.; Wang, Z.; Ding, K. Tetrahedron 2012, 68, 7581.

[101] Zhang, J.; Dong, K.; Wang, Z.; Ding, K. Org. Biomol. Chem. 2012, $10,1598$.

[102] Hou, G.-H.; Xie, J.-H.; Yan, P.-C.; Zhou, Q.-L. J. Am. Chem. Soc. 2009, 131, 1366.

[103] Yan, P.-C.; Xie, J.-H.; Hou, G.-H.; Wang, L.-X.; Zhou, Q.-L. $A d v$. Synth. Catal. 2009, 351, 3243.

[104] Xie, J.-H.; Yan, P.-C.; Zhang, Q.-Q.; Yuan, K.-X.; Zhou, Q.-L. ACS Catal. 2012, 2, 561.

[105] Schmitz, C.; Leitner, W.; Franciò, G. Eur. J. Org. Chem. 2015, 6205 .

[106] Hattori, G.; Hori, T.; Miyake, Y.; Nishibayashi, Y. J. Am. Chem. Soc. 2007, 129, 12930
[107] Li, Y.; Feng, Y.; He, Y. M.; Chen, F.; Pan, J.; Fan, Q.-H. Tetrahedron Lett. 2008, 49, 2878

[108] Pignataro, L.; Lynikaite, B.; Cvengros, J.; Marchini, M.; Piarulli, U.; Gennari, C. Eur. J. Org. Chem. 2009, 2009, 2539.

[109] Pignataro, L.; Carboni, S.; Civera, M.; Colombo, R.; Piarulli, U.; Gennari, C. Angew. Chem., Int. Ed. 2010, 49, 6633

[110] Pignataro, L.; Boghi, M.; Civera, M.; Carboni, S.; Piarulli, U.; Gennari, C. Chem.-Eur. J. 2012, 18, 1383.

[111] Pignataro, L.; Bovio, C.; Civera, M.; Piarulli, U.; Gennari, C. Chem.-Eur. J. 2012, 18, 10368.

[112] Li, Y.; He, Y.-M.; Li, Z.-W.; Zhang, F.; Fan, Q.-H. Org. Biomol. Chem. 2009, 7, 1890.

[113] Wieland, J; Breit, B. Nat. Chem. 2010, 2, 832.

[114] Doherty, S.; Robins, E. G.; Pál, I.; Newman, C. R.; Hardacre, C.; Rooney, D. Tetrahedron: Asymmetry 2003, 14, 1517.

[115] Simons, C.; Hanefeld, U.; Arends, I. W. C. E.; Minnaard, A. J.; Maschmeyer, T.; Sheldon, R. A. Chem. Commun. 2004, 2830.

[116] Wang, X. W.; Ding, K. L. J. Am. Chem. Soc. 2004, 126, 10524.

[117] Hu, X.-P.; Huang, J.-D.; Zeng, Q.-H.; Zheng, Z. Chem. Commun. 2006, 293.

[118] Zhao, Y.; Huang, H.; Shao, J.; Xia, C. Tetrahedron: Asymmetry 2011, 22, 769 .

[119] Escárcega-Bobadilla, M. V.; Rodríguez-Pérez, L.; Teuma, E. Catal. Lett. 2011, 141, 808.

[120] Zhang, X.; Liu, X.; Peng, J.; Zhao, Y.; Yang, Q. Catal. Sci. Technol. 2014, 4,1012 . 